\title{
QUANTUM HALL EFFECT AND QUILLEN METRIC
}

\author{
SEMYON KLEVTSOV ${ }^{1}$, XIAONAN MA ${ }^{2}$, GEORGE MARINESCU ${ }^{3}$ AND PAUL WIEGMANN ${ }^{4}$
}

${ }^{1}$ Universität zu Köln, Mathematisches Institut, Weyertal 86-90, 50931 Köln, Germany

${ }^{2}$ Université Paris Diderot - Paris 7, UFR de Mathématiques, Case 7012, 75205 Paris Cedex 13, France

${ }^{3}$ Universität zu Köln, Mathematisches Institut, Weyertal 86-90, 50931 Köln, Germany EInstitute of Mathematics 'Simion Stoilow', Romanian Academy, Bucharest, Romania

${ }^{4}$ Department of Physics, University of Chicago, 929 57th St, Chicago, IL 60637, USA

sam.klevtsov@gmail.com, xiaonan.ma@imj-prg.fr,

gmarines@math.uni-koeln.de, wiegmann@uchicago.edu

\begin{abstract}
We study the generating functional, the adiabatic curvature and the adiabatic phase for the integer quantum Hall effect (QHE) on a compact Riemann surface. For the generating functional we derive its asymptotic expansion for the large flux of the magnetic field, i.e., for the large degree $k$ of the positive Hermitian line bundle $L^{k}$. The expansion consists of the anomalous and exact terms. The anomalous terms are the leading terms of the expansion. This part is responsible for the quantization of the adiabatic transport coefficients in QHE. We then identify the non-local (anomalous) part of the expansion with the Quillen metric on the determinant line bundle, and the subleading exact part with the asymptotics of the regularized spectral determinant of the Laplacian for the line bundle $L^{k}$, at large $k$. Finally, we show how the generating functional of the integer QHE is related to the gauge and gravitational $(2+1)$ d Chern-Simons functionals. We observe the relation between the Bismut-Gillet-Soulé curvature formula for the Quillen metric and the adiabatic curvature for the electromagnetic and geometric adiabatic transport of the integer Quantum Hall state. Then we relate the adiabatic phase in QHE to the eta invariant and show that the geometric part of the adiabatic phase is given by the Chern-Simons functional.
\end{abstract}

\section{CONTEnTs}

1. Introduction

1.1. Background on QHE

1.2. Mathematical background

1.3. Main results

1.4. Further context

2. Generating functional and determinant line bundle

2.1. Wave functions on the lowest Landau level

2.2. Generating functional

2.3. Determinant line bundle and Quillen metric

3. Large $k$ asymptotics of the generating functional

3.1. Proof of Theorem 1 
4. Regularized spectral determinant of the Laplacian

5. Curvature formula for Quillen metric and adiabatic transport

5.1. Curvature formula and adiabatic transport in QHE

5.2. Proof of Theorem 3

Appendix A: Quillen metric and analytic torsion

Appendix B: Expansion of the Bergman kernel on Riemann surfaces

References

\section{INTRODUCTION}

Quantum Hall effect (QHE) arises as a result of a collective motion of electronic liquid confined to the plane in a quantizing magnetic field. Over the last thirty years, several mathematical models of QHE have been developed. In early important papers [6, 7, 48, 49] a fundamental relation between QHE and the Quillen metric [56] has been used to illuminate quantization of transport coefficients. Since then this relation has not been sufficiently explored. This paper intends to fill this gap. We further develop the relation between Quillen metric and QHE, and demonstrate that the anomaly formula for Quillen metric (sometimes the name "Quillen anomaly" is used for brevity) is the powerful approach to physics and mathematics of QHE and is a particularly useful approach to capture the universal transport coefficients. The relation between the Quillen anomaly and QHE also allows us to obtain the asymptotic expansion of the spectral determinant of the Laplacian in large magnetic field.

The adiabatic transport coefficients are perhaps the most important observables of QHE. They are precisely quantized on $\mathrm{QH}$ plateaus. These coefficients are encoded by the adiabatic curvature 2-form, see e.g., [5]. We will show that the adiabatic curvature 2 -form and through it the quantized transport coefficients, naturally follow from the formula [18] (see also [13, 16]) for the curvature of the determinant line bundle. Also we will show how the curvature formula can be used in order to obtain the geometric part of the adiabatic phase of the QHE in the form of the gauge and gravitational Chern-Simons functional. The geometric part of the adiabatic phase has been recently computed in papers [1, 39, 40], extending the earlier approaches of [37] and [68]. Our method in this paper is based on generating functional (see, e.g., [6, 48, 49, 42, 34, 25, 26, 43. for an incomplete list of references). One of the outcomes of this article is a clarification of the relation between the generating functional QH-state approach, the Quillen anomaly and the Chern-Simons functional. In this paper we limit our studies to the integer QHE.

1.1. Background on QHE. Quantum Hall effect occurs in certain two-dimensional electron systems subjected to a high magnetic field. If the current $J$ is forced through the sample in $x$-direction, the Hall voltage $V_{H}$ is observed in the perpendicular direction $y$ on the plane. The Hall conductance $\sigma_{H}=J / V_{H}$, measured as a function of magnetic field at a fixed chemical potential, follows a series of transitions between the plateaus, where it takes constant rational values $\sigma_{H}=\nu$ in units of $e^{2} / h$ with a very high precision. The case where $\nu \in \mathbb{Z}$ is referred as the integer QHE and $\nu=p / q$ is referred to as the fractional QHE. The former case can be explained by a system of free (non-interacting) electrons and the latter is strongly interacting system (via Coulomb forces). While the mechanism behind the QHE is completely different in the two cases, the quantization of the transport coefficients is explained by invoking their relations to 
certain Chern numbers. This paradigm is traced back to Ref. 64 and was elaborated in more detail in Refs. 6, 7] and [4, 49. Let us briefly recall the main points of their construction.

The idea is to consider the QHE on a compact Riemann surface $\Sigma$, where the collective wave function $\Psi\left(z_{1}, \ldots, z_{N}\right)$ describing the electrons naturally acquires dependence on the parameters associated with the geometry of $\Sigma$. To give an example, we need to recall that in quantum mechanics of particle in a magnetic field, the wave function is a section of an appropriate line bundle $\mathrm{L}$ on $\Sigma$, see e.g. 32 for a review. The flat component (called $L_{\varphi}$ in what follows) of the line bundle $\mathrm{L}$ is parameterized by coordinates $\left(\varphi_{1}^{a}, \varphi_{2}^{a}\right) \in T^{2 \mathrm{~g}}$ in the Jacobian torus (see Eq. (42) for more detail), which physically correspond to Aharonov-Bohm (AB) solenoid fluxes, piercing through the holes of the surface (for genus $\mathrm{g}>0$ ). The wave function $\Psi\left(z_{1}, \ldots, z_{N} \mid \varphi^{a}\right.$ ) now depends on $\varphi^{a}$, which can be varied adiabatically with time $t$ and we can define adiabatic (Berry) connection

$$
\mathcal{A}=i\left\langle\Psi, d_{t} \Psi\right\rangle_{L^{2}}
$$

and curvature $\Omega=\left\langle d_{t} \Psi, d_{t} \Psi\right\rangle_{L^{2}}$, see Ref. [60]. The current $J_{a}$ around the ath flux is equal $J_{a}=i \omega_{a b} \dot{\varphi}^{b}$, where $\omega_{a b}$ are coefficients of the conductance two-form $\omega$ on $T^{2 \mathrm{~g}}$, computed via Quillen anomaly arguments in Ref. [7],

$$
\omega=\Omega+d d^{c} \log \operatorname{det} \Delta_{\mathrm{L}}, \quad \Omega=\sigma_{H} \sum_{a=1}^{\mathrm{g}} d \varphi_{1}^{a} \wedge d \varphi_{2}^{a},
$$

where $\Delta_{\mathrm{L}}$ is the Laplacian for the line bundle L, defined in (40). Thus the Hall conductance $\sigma_{H}$, which is the coefficient in the conductance 2 -form $\Omega$ in (2) is quantized since $[\Omega] \in 2 \pi \mathbb{Z}$, and $d d^{c} \log$ det $\Delta_{\mathrm{L}}$ can be interpreted as the mesoscopic fluctuations around the quantized value of $\sigma_{H}$. Mesoscopic fluctuations vanish exponentially with the size of the system and its average over the ensemble of systems with respect to remaining parameters identically vanishes.

In this paper we focus on the adiabatic geometric transport on the moduli space of complex structures on $\Sigma$, which gives rise to novel quantized transport coefficients [7, 48, 49, 43]. In this paper we explore this program further, along various lines. Before stating the results, we introduce the main objects of interest.

1.2. Mathematical background. Consider a positive Hermitian holomorphic line bundle $L$ with a Hermitian metric $h$, and its $k$ th tensor power $\left(L^{k}, h^{k}\right)$, on a compact (connected) Riemann surface $\Sigma$ of genus g and the space of global holomorphic sections $H^{0}\left(\Sigma, L^{k}\right)$. Physically, the basis of the vector space $H^{0}\left(\Sigma, L^{k}\right)$ corresponds to the states on the lowest Landau level (LLL). We then tensor the line bundle $\left(L^{k}, h^{k}\right)$ with the $s$-th tensor power $K^{s}$ of the canonical line bundle $K$ on $\Sigma$, where $s \in \frac{1}{2} \mathbb{Z}$ is called the spin. Note that the canonical bundle $K$ on a Riemann surface admits a square root, namely, there exists a holomorphic line bundle $\mathcal{K}$ such that $\mathcal{K}^{2}=K$, so that $K^{s}:=\mathcal{K}^{2 s}$ makes sense for $s \in \frac{1}{2} \mathbb{Z}$.

For the line bundle $L^{k}$ the positive integer $k$ has a meaning of total flux of the magnetic field $\frac{1}{2 \pi} \int_{\Sigma} F=k$, where $-i F=F_{z \bar{z}} d z \wedge d \bar{z}, F_{z \bar{z}}=-\partial_{z} \partial_{\bar{z}}\left(\log h^{k}\right)$, is the curvature $(1,1)$-form of $L^{k}$ and we assume that $F_{z \bar{z}}>0$ everywhere on $\Sigma$. Writing the Riemannian metric as $d s^{2}=2 g_{z \bar{z}}|d z|^{2}$ in local complex coordinates $z, \bar{z}$, the Hermitian metric on $K^{s}$ is $\left(g_{z \bar{z}}\right)^{-s}$. Then $B=g^{z \bar{z}} F_{z \bar{z}}$ is the magnetic field. The curvature form on $K^{s}$ is given by si $\operatorname{Ric}(g)$, where the Ricci form is $\operatorname{Ric}(g)=-\partial_{z} \partial_{\bar{z}}\left(\log g_{z \bar{z}}\right) i d z \wedge d \bar{z}$. Thus $\operatorname{deg} K^{s}=-s \chi(\Sigma)$ and according to the Riemann-Roch formula (25) the dimension of the vector space $H^{0}\left(\Sigma, L^{k} \otimes K^{s}\right)$ of holomorphic sections (LLL 
states) equals

$$
N_{k}=\operatorname{dim} H^{0}\left(\Sigma, L^{k} \otimes K^{s}\right)=k+(1-\mathrm{g})(1-2 s),
$$

assuming $\operatorname{deg} L^{k} \otimes K^{s}>\operatorname{deg} K$. Since the physics of QHE involves a large number of particles, we will always assume that the flux of the magnetic field is large $k \gg 1$, while $s$ is kept fixed, so that the dimension $N_{k}$ is also large.

Consider now a basis $s_{j}(z), j=1, \ldots N_{k}$, of the space of holomorphic sections $H^{0}\left(\Sigma, L^{k} \otimes K^{s}\right)$. The positive measure on configurations of $N_{k}$ electrons derived from QH-state is defined as follows:

Definition 1. Consider the following finite measure on a configuration of $N_{k}$ points

$$
\left|\Psi\left(z_{1}, \ldots, z_{N_{k}}\right)\right|^{2} \prod_{j=1}^{N_{k}} \sqrt{g} d^{2} z_{j}:=\frac{1}{N_{k} !}\left|\operatorname{det} s_{i}\left(z_{j}\right)\right|^{2} \prod_{j=1}^{N_{k}} h^{k}\left(z_{j}, \bar{z}_{j}\right) g_{z \bar{z}}^{-s}\left(z_{j}, \bar{z}_{j}\right) \sqrt{g} d^{2} z_{j} .
$$

The partition function $Z_{k}$ is defined as

$$
Z_{k}=\frac{1}{(2 \pi)^{N_{k}}} \int_{\Sigma^{N_{k}}}\left|\Psi\left(z_{1}, \ldots, z_{N_{k}}\right)\right|^{2} \prod_{j=1}^{N_{k}} \sqrt{g} d^{2} z_{j},
$$

and $\log Z_{k}$ is called the generating functional.

By construction $Z_{k}$ depends on the choice of the basis in $H^{0}\left(\Sigma, L^{k} \otimes K^{s}\right)$, which we specify in a moment, on the Riemannian metric $g$ on $\Sigma$, the Hermitian metric $h^{k}$ on $L^{k}$, and is invariant if we transform the defining data by a diffeomorphism. Also, $Z_{k}$ implicitly depends on the choice of a complex structure $J$ on $\Sigma$ and a point in Jacobian variety. The latter is because once we have chosen the line bundle $L^{k}$, all other choices are related to by tensoring $L^{k} \otimes L_{\varphi}$ with a flat line bundle $L_{\varphi}$ (which is trivial as a smooth line bundle, but non-trivial as holomorphic line bundle). Following standard notations we denote by $\mathcal{M}_{\mathrm{g}}$ of complex structures on the surface $\Sigma$ of genus g and by $\operatorname{Jac}(\Sigma)$ the moduli space of flat line bundles on $\Sigma$. Then $Z_{k}$ varies over the parameter space (complex manifold),

$$
Y=\mathcal{M}_{\mathrm{g}} \times \operatorname{Jac}(\Sigma) .
$$

Note that the complex structure on the fiber $\operatorname{Jac}\left(\Sigma_{b}\right)$ depends on $b \in \mathcal{M}_{\mathrm{g}}$. For each $y \in Y$ we denote by $\Sigma_{y}$ the Riemann surface corresponding to $y$, and we have a corresponding partition function $Z_{k}(y)$. As we have already mentioned in \$1.1 physically the Jacobian variety $\operatorname{Jac}(\Sigma)$ corresponds to the space of Aharonov-Bohm solenoid fluxes.

The dependence of generating functional on $y \in Y$ encodes the adiabatic transport coefficients related to electromotive $(\operatorname{Jac}(\Sigma))$ and geometric $\left(\mathcal{M}_{\mathrm{g}}\right)$ transport, as we now explain. Consider the holomorphic line bundle $\mathcal{L}=\operatorname{det} H^{0}\left(\Sigma, L^{k} \otimes K^{s}\right)$ over $Y$, called determinant line bundle, with fiber $\mathcal{L}_{y}=\operatorname{det} H^{0}\left(\Sigma_{y}, L_{y}^{k} \otimes K_{y}^{s}\right)$ for $y \in Y$. For each point $y_{0} \in Y$ there exist a neighborhood $U \subset Y$ of $y_{0}$ and a basis $s_{j}=s_{j}(\cdot, y), j=1, \ldots, N_{k}$, of $H^{0}\left(\Sigma_{y}, L_{y}^{k} \otimes K_{y}^{s}\right)$ holomorphically varying with $y \in U$. We obtain thus a local holomorphic frame

$$
\mathcal{S}: U \rightarrow \mathcal{L}, \quad y \mapsto \mathcal{S}(y):=s_{1}(\cdot, y) \wedge \cdots \wedge s_{N_{k}}(\cdot, y) \in \mathcal{L}_{y} .
$$

This is the holomorphic component of the state $\Psi$ in Eq. (3), which also varies holomorphically on the parameter space $Y$. We define now $Z_{k}(y)$ Eq. (44) for $y \in U$ as the square of the $L^{2}$-norm of $\mathcal{S}(y)$ with help of $s_{j}(\cdot, y)$, by taking the pointwise norm of $s_{1}(\cdot, y) \wedge \cdots \wedge s_{N_{k}}(\cdot, y)$ as a section of $\left(L_{y}^{k} \otimes K_{y}^{s}\right) \otimes \ldots \otimes\left(L_{y}^{k} \otimes K_{y}^{s}\right) \rightarrow \Sigma_{y}^{N_{k}}$ and integrating over $\Sigma_{y}^{N_{k}}$. In Lemma 1 (Section 5) we 
show that the adiabatic curvature $(1,1)$-form $\Omega$ on $Y$ is given by the curvature of the Chern connection on $\mathcal{L}$ endowed with the $L^{2}$-metric,

$$
\Omega=-\partial_{Y} \bar{\partial}_{Y} \log Z_{k}
$$

where $\bar{\partial}_{Y}$ is the Cauchy-Riemann operator on $Y$ and $\partial_{Y}$ is defined by the decomposition $d_{Y}=$ $\partial_{Y}+\bar{\partial}_{Y}$ of the exterior derivative $d_{Y}$ on $Y$. In local complex coordinates $\left(y_{j}, \bar{y}_{j}\right)$ on $Y, \partial_{Y}=$ $\sum_{j} d y_{j} \wedge \partial_{y_{j}}, \bar{\partial}_{Y}=\sum_{j} d \bar{y}_{j} \wedge \partial_{\bar{y}_{j}}$, where $\partial_{y_{j}}=\partial / \partial y_{j}, \partial_{\bar{y}_{j}}=\partial / \partial \bar{y}_{j}$. Therefore the generating functional $\log Z_{k}$ plays the role of a Kähler potential on $Y$. Note that there is a freedom in the definition of $Z_{k}$, since we can always multiply $Z_{k} \rightarrow\left|f(y)^{2}\right| Z_{k}$, where $f(y)$ is a nonvanishing holomorphic function on $Y$. In particular, $Z_{k}$ is not necessary modular invariant on $\mathcal{M}_{\mathrm{g}}$. However, this ambiguity will be localized at the boundary of $Y$ and will not contribute to the adiabatic curvature Eq. (77) in the bulk, where the results of this paper apply. Additional arguments, similar to that of Ref. [8], can be invoked to fix this ambiguity, which we do not consider here. The integrals of $\Omega$ over smooth closed two-cycles in $Y$ define quantized adiabatic transport coefficients. Here we observe the following relation

$$
\Omega=\Omega^{\mathcal{L}}-\partial_{Y} \bar{\partial}_{Y} \operatorname{det}^{\prime} \Delta_{\mathrm{L}},
$$

between the adiabatic curvature $\Omega$, the regularized spectral determinant of the (Kodaira) Laplacian $\Delta_{\mathrm{L}}=\bar{\partial}_{\mathrm{L}}^{*} \bar{\partial}_{\mathrm{L}}$ for the line bundle $\mathrm{L}=L^{k} \otimes K^{s}$ (the asterisk in $\bar{\partial}_{\mathrm{L}}^{*}$ denotes the adjoint operator) and the curvature of the Quillen metric $\Omega^{\mathcal{L}}$, to be defined next. Since $\partial_{Y} \bar{\partial}_{Y} \log \operatorname{det}^{\prime} \Delta_{\mathrm{L}}$ is an exact form on $Y$, (8) shows that $\Omega$ and $\Omega^{\mathcal{L}}$ belong to the same de Rham cohomology class.

Following Quillen [56] we define a smooth Hermitian metric on $\mathcal{L}$, called Quillen metric, such that the norm squared of the section $\mathcal{S}$ is given by Eq. (43),

$$
\|\mathcal{S}\|^{2}=\frac{\operatorname{det}\left\langle s_{j}, s_{i}\right\rangle_{L^{2}}}{\operatorname{det}^{\prime} \Delta_{\mathrm{L}}}
$$

where $L^{2}$ metric is given by Eqns. (29), (30), since from the determinant formula (33), it follows that $\operatorname{det}\left\langle s_{j}, s_{i}\right\rangle_{L^{2}}=Z_{k}$. By general theory, the Chern curvature of the Quillen metric is given on $U$ by

$$
\Omega^{\mathcal{L}}=-\partial_{Y} \bar{\partial}_{Y} \log \|\mathcal{S}\|^{2}
$$

The curvature of the Quillen metric is then the following $(1,1)$-form on $Y$,

$$
\Omega^{\mathcal{L}}=-\partial_{Y} \bar{\partial}_{Y} \log \frac{Z_{k}}{\operatorname{det}^{\prime} \Delta_{\mathrm{L}}}
$$

and the relation (8) follows immediately.

Quillen [56] observed that the metric in (9) is a smooth metric on the determinant line bundle $\mathcal{L} \rightarrow Y$, where $Y$ is the space of holomorphic structures of a complex vector bundle on a fixed Riemann surface and computed precisely the curvature of its Chern connection. Adapting Quillen's result yields the formula for the curvature $\Omega^{\mathcal{L}}$ in the case of $Y=\operatorname{Jac}(\Sigma)$. If $Y=\mathcal{M}_{\mathrm{g}}$ is the moduli space for Riemann surfaces, $\Omega^{\mathcal{L}}$ was considered by Belavin-Knizhnik [8], and for a smooth family of Dirac operators by Bismut-Freed [16] and (for higher-dimensional Kähler manifolds) by Bismut-Gillet-Soulé [18], see also [2] for the account in physics literature. Note that in general the cohomology class of $\Omega^{\mathcal{L}}$ is non-trivial, as a consequence of Atiyah-Singer family index theorem [4].

The adiabatic phase, or holonomy $\exp \left(-\int_{\mathcal{C}} \mathcal{A}^{\mathcal{L}}\right)$ is the phase factor acquired by the wave function under the transport along a smooth closed contour $\mathcal{C}$ in $Y$. The adiabatic phase 
consist of two distinct parts: the topological part and the geometric part. The topological part, arises if the contour $\mathcal{C}$ is non-contractible, such as e.g., the Dehn twists on the torus, and is independent of the smooth variations of the contour. Such contour encloses a boundary point in $Y$ where the holomorphic function $f(y)$ discussed above is singular. This part is due to a flat connection on $\mathcal{L} \rightarrow Y$, which has a non-zero holonomy. The geometric part of the adiabatic phase arises even if the contour is contractible and depends on the shape of the contour. The sum of two phases equal full adiabatic phase, and if the contour is contractible, the geometric phase is the total phase.

Next, following (8), we will consider the connection 1-form, associated to the curvature of the Quillen metric $\Omega^{\mathcal{L}}=d_{Y} \mathcal{A}^{\mathcal{L}}$. Then $\mathcal{A}^{\mathcal{L}}-\mathcal{A}=\frac{1}{2}\left(\bar{\partial}_{Y}-\partial_{Y}\right) \log \operatorname{det}^{\prime} \Delta_{\mathrm{L}}$ is a globally defined differential form on $Y$, related to the Bismut-Cheeger eta form [15, Definition 4.33], [16, (3.166)], [17, Theorem 2.20]. The holonomy $\exp \left(-\int_{\mathcal{C}} \mathcal{A}^{\mathcal{L}}\right)$ on a closed contour $\mathcal{C}$ is related to the $\eta$-invariant [3, 70], [16, Theorem 3.16], [14, §6]. In Th. 3 we will compute the part of the geometric adiabatic phase $\int_{\mathcal{C}} \mathcal{A}^{\mathcal{L}}$, associated to the Quillen metric.

One of the central goals of this article is to determine the large $k$ asymptotics of the generating functional $\log Z_{k}$, to determine its dependence on the geometric parameters and relate it to the asymptotics of the Quillen metric and to compute the transport coefficients and the geometric adiabatic phases. As a consequence of Eq. (9), we also obtain the asymptotics of the regularized spectral determinant and compare it to the previous results of Bismut-Vasserot [20].

1.3. Main results. The first result concerns the large $k$ asymptotic expansion of the generating functional $\log Z_{k}$. We show that the asymptotic expansion has schematically the form $\log Z_{k}=$ $\log Z_{H}+\mathcal{F}$, where $\log Z_{H}$ is the non-local (also referred to as "anomalous") part of the expansion, and the "exact part" $\mathcal{F}$ contains local terms. The anomalous part is completely defined by the Quillen metric (9).

Consider the metrics $g_{0}, g$ and $h_{0}^{k}, h^{k}$, related as $g=g_{0}+\partial_{z} \partial_{\bar{z}} \phi$ and $h^{k}=h_{0}^{k} e^{-k \psi}$, for scalar functions $\phi, \psi \in \mathcal{C}^{\infty}(\Sigma)$ cf. (35), (36). We study the large $k$ asymptotics of $\log \frac{Z_{k}}{Z_{k 0}}$, where $Z_{k 0}$ is defined as in Eq. (44) using the metrics $g_{0}$ and $h_{0}^{k}$.

THEOREM 1 (\$3.1). At large $k$ the following asymptotic expansion holds

$$
\log \frac{Z_{k}}{Z_{k 0}}=\log \frac{Z_{H}}{Z_{H 0}}+\mathcal{F}-\mathcal{F}_{0},
$$

where the anomalous part $\log Z_{H}$ is given by

$$
\begin{aligned}
\log \frac{Z_{H}}{Z_{H 0}}= & \frac{2}{\pi} \int_{\Sigma}\left(\left(A_{z}+\frac{1-2 s}{2} \omega_{z}\right)\left(A_{\bar{z}}+\frac{1-2 s}{2} \omega_{\bar{z}}\right)-\frac{1}{12} \omega_{z} \omega_{\bar{z}}\right) d^{2} z \\
& -\left(A \rightarrow A_{0}, \omega \rightarrow \omega_{0}\right),
\end{aligned}
$$

(see also Eq. (51) for another form of this equation), where the notation $\left(A \rightarrow A_{0}, \omega \rightarrow \omega_{0}\right)$ just means that we replace $A$ by $A_{0}$ and $\omega$ by $\omega_{0}$ in the previous expression, and the exact part $\mathcal{F}$ admits a large $k$ asymptotic expansion, with the first three terms given by

$$
\mathcal{F}=-\frac{1}{2 \pi} \int_{\Sigma}\left[\frac{1}{2} B \log \frac{B}{2 \pi}+\frac{2-3 s}{12} R \log \frac{B}{2 \pi}+\frac{1}{24}(\log B) \Delta_{g}(\log B)\right] \sqrt{g} d^{2} z+\mathcal{O}(1 / k),
$$

where $\Delta_{g}=2 g^{z \bar{z}} \partial_{z} \partial_{\bar{z}}$ is the scalar Laplacian.

Moreover, 
TheOREM 2 (\$4). The anomalous part of the generating functional corresponds to the Quillen metric (9),

$$
\log Z_{H}=\log \|\mathcal{S}\|^{2}
$$

and the exact part corresponds to the spectral determinant

$$
\log \frac{\operatorname{det}^{\prime} \Delta_{\mathrm{L}}}{\operatorname{det}^{\prime} \Delta_{\mathrm{L} 0}}=\mathcal{F}-\mathcal{F}_{0} .
$$

Let us first explain the content of Eqs. (11), (12). Here $A_{z}, A_{\bar{z}}$ are the components of the connection 1-form for the magnetic field strength $F=d A$, and $\omega_{z}, \omega_{\bar{z}}$ are the components of the spin connection 1-form $\operatorname{Ric}(g)=d \omega$, see (19), (21) for details. Also, $A_{0}$ and $\omega_{0}$ refer to the same objects defined with respect to the background metrics $g_{0}$ and $h_{0}^{k}$. The objects are weighted as follows: $B$ (and $A_{z}, A_{\bar{z}}$ ) are of order $k$, meaning $B / k$ is a smooth positive function on $\Sigma$, independent of $k$, while the objects that depend on the metric, such as $\Delta_{g}, R$ and $\omega_{z}, \omega_{\bar{z}}$ are considered to be of order 1 . Hence the remainder $1 / k$ terms in (12) may contain terms of the type $1 / B$.

The proof of Theorem 1 is based on the known asymptotic expansion of the Bergman kernel. The variational formula (56) connects the large $k$ asymptotic expansion of $\log Z_{k}$ with the large $k$ asymptotic expansion of the Bergman kernel for positive Hermitian holomorphic line bundle $L^{k}$, established in Refs. [73, 27]. The more general version of the Bergman kernel expansion pertinent to the present paper was given in Ref. [51,52]. We also refer to [51] for a comprehensive study of several analytic and geometric aspects of Bergman kernel. Suppose $\left\{s_{j}^{\prime}\right\}$ is a basis of $H^{0}\left(\Sigma, L^{k} \otimes K^{s}\right)$, orthonormal with respect to the inner product Eq. (30). Then the Bergman kernel on the diagonal $B_{k}(z, \bar{z})$ is defined by the sum

$$
B_{k}(z, \bar{z})=\sum_{j=1}^{N_{k}}\left|s_{j}^{\prime}(z)\right|^{2} h^{k} g_{z \bar{z}}^{-s},
$$

The first three terms of its large $k$ asymptotic expansion of the Bergman kernel on the diagonal are

$$
B_{k}(z)=B+\frac{1-2 s}{4} R+\frac{1}{4} \Delta_{g} \log B+\mathcal{O}(1 / k) .
$$

In Eq. (57) we list more terms in this expansion, relevant for the result of Theorem 1, and in the Appendix B we review the Bergman kernel expansion.

Integrating the variational formula (56), we find that the generating functional can be separated in two parts. The anomalous part (11) is a non-local functional of the magnetic field density $B$ and scalar curvature $R$. This part is given in Eq. (11). The exact part $\mathcal{F}$ of the asymptotic expansion consists of local functionals of $B$ and $R$. Using Theorem 2 we interpret $\mathcal{F}$ as $\log \operatorname{det}^{\prime} \Delta_{\mathrm{L}}$ and explain that all corrections of order $1 / k$ to $\mathcal{F}$ are in fact given by local functionals.

Then, in Theorem 2 we identify the anomalous part of the expansion $\log Z_{H}$ with the Quillen metric (9). We use the heat kernel formula for the regularized determinant $\operatorname{det}^{\prime} \Delta_{\mathrm{L}}$ of the Laplacian for the line bundle $\mathrm{L}=L^{k} \otimes K^{s}$, in order to derive the anomaly formula for $\log \|\mathcal{S}\|^{2}$ under the variations of Kähler and magnetic potentials. Let us point out that this is consistent with the general anomaly formula for $\log \|\mathcal{S}\|^{2}$ of Ref. [18, Theorem 1.23]. As a consequence, we note that the exact part $\mathcal{F}(12)$ of the expansion can be identified with asymptotics of 
$\log \operatorname{det}^{\prime} \Delta_{\mathrm{L}}$ for high powers $k$ of the positive line bundle. The leading term in this expansion was derived by Bismut-Vasserot [20], see also [51, \$5.5.5]. Thus Eq. (12) provides the leading corrections to their result.

Theorem 1 generalizes the large $k$ asymptotic expansion of the generating functional derived in Ref. [42, where the case of constant magnetic field $B=k$ and $s=0$ was considered. For analogous results in fractional QHE, derived by various physics methods see Refs. [72, 25, 26, 34]. In particular the exact terms in Eq. (12) are in agreement with [26, Eq. (130)].

In the last section we derive the formula for the geometric part of the adiabatic phase, associated with the curvature form $\Omega^{\mathcal{L}}$. The starting point is the Bismut-Gillet-Soule curvature Eq. (87)] $]^{1}([18$, Theorems 1.9, 1.27], see also [16, 22])

$$
\Omega^{\mathcal{L}}=-2 \pi i \int_{M \mid Y}[\operatorname{Ch}(E) \operatorname{Td}(T M \mid Y)]_{(4)} .
$$

Here $\sigma: M \rightarrow Y$ is a fibration over $Y$ (universal curve), with fiber diffeomorphic to $\Sigma$, and $\Sigma_{y}=\sigma^{-1}(y)$ for $y \in Y$. Also,

$$
E=\tilde{L}^{k} \otimes \tilde{K}^{s} \rightarrow M,
$$

where the line bundles $\tilde{L}^{k}, \tilde{K}^{s}$ have been extended from the individual Riemann surfaces $\Sigma_{y}$ to the universal curve, see $\$ 5$ for details. Moreover, $\mathrm{Ch}(E)$ is the Chern form and $\operatorname{Td}(T M \mid Y)$ is the Todd form and the integrand in Eq. (14) is a 4-form on the manifold $M$. The integration goes over the fiber $\Sigma_{y}$ over a fixed point $y \in Y$, so that the result is a 2 -form on $Y$. We introduce the curvature $\mathrm{F}^{E}=\mathrm{F}-s \mathrm{R}_{T M \mid Y}$ where $\mathrm{F}$ refers to the part of the curvature 2-form corresponding to the line bundle $\tilde{L}^{k} \rightarrow M$. Locally on $M, \mathrm{~F}=d A$, with a connection 1 -form $A$, and $\mathrm{R}_{T M \mid Y}=d \omega$ with a spin-connection 1-form $\omega$.

Then using the curvature formula (14) we show that the geometric part (induced by $\Omega^{\mathcal{L}}$ ) of the adiabatic phase is given by the Chern-Simons action.

THEOREM 3 (\$5.2). The adiabatic phase arising from the Quillen metric over a closed contour $\mathcal{C} \subset Y$ takes the form

$$
\exp \left(-\int_{\mathcal{C}} \mathcal{A}^{\mathcal{L}}\right)=\exp \left(2 i \pi \int_{\sigma^{-1}(G)}[\operatorname{Ch}(E) \operatorname{Td}(T M \mid Y)]_{(4)}\right)
$$

if $\mathcal{C}$ is the boundary of a domain $G \subset Y$.

For a general closed contour $\mathcal{C} \subset Y$, if the metric $g$ on $T \Sigma$ is the restriction of a Kähler metric on a neighborhood of $\sigma^{-1}(\mathcal{C})$, the adiabatic phase is given by

$$
\exp \left(-\int_{\mathcal{C}} \mathcal{A}^{\mathcal{L}}\right)=\exp (2 i \pi \bar{\eta})
$$

where $\bar{\eta}$ is the adiabatic limit of the reduced eta invariant for the fibration $\sigma: \sigma^{-1}(\mathcal{C}) \rightarrow \mathcal{C}$. If $\bar{\eta}_{1}$ is the adiabatic limit of the reduced eta invariant associated to metrics $g_{1}$ and $h_{1}^{L}$ on $T \Sigma$ and $L$, the variation of the adiabatic limit is given by the $(2+1) d$ Chern-Simons functional

$$
\begin{array}{r}
2 \pi i\left(\bar{\eta}-\bar{\eta}_{1}\right)=\frac{i}{4 \pi} \int_{\sigma^{-1}(\mathcal{C})} A \wedge d A+\frac{1-2 s}{2}(A \wedge d \omega+d A \wedge \omega)+\left(\frac{(1-2 s)^{2}}{4}-\frac{1}{12}\right) \omega \wedge d \omega \\
-\left(A \rightarrow A_{1}, \omega \rightarrow \omega_{1}\right) .
\end{array}
$$

\footnotetext{
${ }^{1}$ In the QHE context this formula was invoked in Ref. 63].
} 
For a general contour $\mathcal{C}$ and general metric $g$ we can obtain a similar formula to (17) by using the anomaly formula [18, Theorem 1.23] (cf. Theorem 4, (66) ) as in the proof of [18, Theorem 1.27 .

For $s=0$ this formula is equivalent to the Chern-Simons action of QHE derived in Ref. 11. Let us also point out that results of this paper admit generalization to the Laughlin states in fractional QHE with the filling fraction $\nu=1 / \beta$, where $\beta$ is an integer, as was reported in Ref. [43. These " $\beta$-deformations" of the Quillen anomaly and Bismut et. al. curvature formulas will be explored further elsewhere.

1.4. Further context. It was understood early on that the exact quantization of the transport coefficients in QHE essentially has a geometric origin [64, 54, 5, 6, 7]. In particular, the quantization of the Hall conductance in the integer QHE case was attributed to the fact, that the $\mathrm{QH}$-wave functions are sections of a line bundle over the space of Aharonov-Bohm fluxes (Jacobian variety $J a c(\Sigma)$ ) on a higher-genus Riemann surface, and the corresponding adiabatic curvature belongs to an integer Chern class [6]. In the seminal papers [7] and [48] the existence of another quantized parameter in the integer QHE - so called odd viscosity (terms anomalous viscosity or Hall viscosity are also used) - was deduced when considering the adiabatic curvature of integer QH-states for the adiabatic transport on the moduli space of complex structures of a torus. For the moduli spaces of complex structures of higher-genus Riemann surfaces the adiabatic curvature in the integer QHE was first derived in the pioneering paper [49. These results were also generalized to the fractional Quantum Hall states, for the quantization of the conductance [5, 62] and for the anomalous viscosity on the torus [65, 66, 58, 59]. Existence of yet another, third quantized coefficient in both, integer and the fractional QHE associated with complex structure moduli spaces of higher-genus Riemann surfaces was proposed in [43], see [24] for related results.

As we have emphasised, many aspects of the geometry of Quantum Hall states are encoded in the generating functional $\log Z_{k}$ (4). The geometric definition of $Z_{k}$ in QHE context in terms of holomorphic sections of the line bundle and Kähler metrics on the Riemann surface was given in [42. There exist several approaches to calculate the generating functional. In [72] and [25, 26] the Ward identity method was applied in case when $\Sigma$ is the sphere. In [42], as well as in the present work, the large $k$ expansion is obtained from asymptotic expansion of the Bergman kernel [73, 27, 51] on the surface of any genus. Interestingly, objects closely related to the QHE generating functional are useful in Kähler geometry [31, 10, 11]. The field theory approach to calculate the large $k$ asymptotics of the generating functional was developed in [34, and the collective field theory approach was proposed in [46].

Alternatively, in the case of the integer QHE the transport coefficients can be also directly computed through the linear response theory as it has been done in [1]. The linear response theory can be also cast in the form of the so-called effective action approach. Although conceptually different, the effective action of the LLL and the geometric adiabatic phase discussed in this paper are identical in the case of the QHE. Both are given by the Chern-Simons functional. For other relevant approaches to the effective action which also lead to the Chern-Simons functional see [40, 61, 23, 24].

The generating functional is formally equivalent to thermodynamic potential of $2 \mathrm{~d}$ Coulomb plasma of equal charges in a neutralized background, the so-called plasma analogy. From this point of view the normalization factor is a partition function of the $2 \mathrm{~d}$ Coulomb gas (see, e.g., 
[36] for the comprehensive account). This relation gives allows us to establish the link between the thermodynamics of $2 \mathrm{~d}$ Coulomb plasma, Quillen metric and the spectral determinant of the Laplacian.

Finally, let us remark that the most notable occurrence of the Quillen metric in physics goes back to the work of Belavin-Knizhnik [8] on the holomorphic anomaly of the Polyakov string. Cancellation of the holomorphic anomaly yields the result that the measure of the critical string is a modulus square of the holomorphic function on the moduli space. Interestingly, in contrast to the critical string, in QHE the curvature of Quillen metric does not vanish and furthermore encodes important physical information (quantized adiabatic transport).

Acknowledgments. We would like to thank J.-M. Bismut, V. Pestun and S. Zelditch for useful discussions and comments on the manuscript and the anonymous referees for useful comments and suggestions. We thank Zhenghan Wang for pointing us Ref. [41. SK is partially supported by the Max Delbrück prize for junior researchers at the University of Cologne, the Humboldt postdoctoral fellowship and the grants NSh-1500.2014.2, RFBR 15-01-04217 and NSF DMR-1206648. XM is partially supported by ANR-14-CE25-0012-01 and funded through the Institutional Strategy of the University of Cologne within the German Excellence Initiative. GM was partially supported by the DFG project SFB TR12. The research of PW was carried out at the RAS Institute for Information Transmission Problems under support by a grant from the Russian Foundation for Sciences (project 14-50-00150). SK, XM and GM acknowledge the hospitality of the Simons Center for Geometry and Physics where the research leading to this paper has been started.

\section{Generating functional and Determinant line Bundle}

2.1. Wave functions on the lowest Landau level. We begin by defining the wave functions on the lowest Landau level, on a compact (connected) Riemann surface $\Sigma$. Let $(L, h)$ be a positive Hermitian holomorphic line bundle and let $\left(L^{k}, h^{k}\right)$ be its $k$ th tensor power, with $k$ a positive integer. In local complex coordinates $z, \bar{z}$ the metric on $\Sigma$ is diagonal $d s^{2}=2 g_{z \bar{z}}|d z|^{2}$ and the area of the surface will be fixed $\int_{\Sigma} \sqrt{g} d^{2} z=2 \pi$. The curvature $(1,1)$-form of the Hermitian metric is $-i F$, where

$$
F=\left(\partial_{z} A_{\bar{z}}-\partial_{\bar{z}} A_{z}\right) d z \wedge d \bar{z}=-\partial_{z} \partial_{\bar{z}}\left(\log h^{k}\right) i d z \wedge d \bar{z}=: F_{z \bar{z}} i d z \wedge d \bar{z} .
$$

Here $A=A_{z} d z+A_{\bar{z}} d \bar{z}$ is the local gauge connection 1-form for $F$ and

$$
A_{z}=\frac{1}{2} i \partial_{z} \log h^{k}, \quad A_{\bar{z}}=-\frac{1}{2} i \partial_{\bar{z}} \log h^{k} .
$$

The positivity of $(L, h)$ means that $F$ is a positive $(1,1)$-form on $\Sigma$, or equivalently, the function $F_{z \bar{z}}=-\partial_{z} \partial_{\bar{z}} \log \left(h^{k}\right)$ is positive. Instead of the $(1,1)$-form $F$ we will mainly use the magnetic field $B=g^{z \bar{z}} F_{z \bar{z}}$. It is a globally defined scalar function, also everywhere positive on $\Sigma$. Then the integer tensor power $k$ has the meaning of the total flux of the magnetic field through the surface

$$
\frac{1}{2 \pi} \int_{\Sigma} F=\frac{1}{2 \pi} \int_{\Sigma} B \sqrt{g} d^{2} z=k
$$


Let us also introduce the spin-connection 1-form $\omega$, related to the Ricci $(1,1)$-form $\operatorname{Ric}(g)$ as follows

$$
\operatorname{Ric}(g)=R_{z \bar{z}} i d z \wedge d \bar{z}=\left(\partial_{z} \omega_{\bar{z}}-\partial_{\bar{z}} \omega_{z}\right) d z \wedge d \bar{z}
$$

and

$$
\omega_{z}=\frac{1}{2} i \partial_{z} \log g_{z \bar{z}}, \quad \omega_{\bar{z}}=-\frac{1}{2} i \partial_{\bar{z}} \log g_{z \bar{z}} .
$$

The scalar curvature $R$ is given by

$$
R=2 g^{z \bar{z}} R_{z \bar{z}}=-2 g^{z \bar{z}} \partial_{z} \partial_{\bar{z}} \log \sqrt{g}=-\Delta_{g} \log \sqrt{g},
$$

where $\sqrt{g}=2 g_{z \bar{z}}$ and the scalar Laplacian is $\Delta_{g}=2 g^{z \bar{z}} \partial_{z} \partial_{\bar{z}}$. We use the standard normalization for the scalar curvature, so that the Euler characteristic of $\Sigma$ is given by $\chi(\Sigma)=\frac{1}{4 \pi} \int_{\Sigma} R \sqrt{g} d^{2} z$.

The LLL wave functions are in one-to-one correspondence with the holomorphic sections of the line bundle. In our case the line bundle will be the tensor product $\mathrm{L}=L^{k} \otimes K^{s}$, where $K$ is canonical line bundle. The Hermitian metric on the sections of $K^{s}$ is $g_{z \bar{z}}^{-s}$ and its curvature form is $i s \operatorname{Ric}(g)$. The holomorphic sections are solutions of the $\bar{\partial}$-equation

$$
\bar{\partial}_{\mathrm{L}} s(z)=0
$$

where the $\bar{\partial}$-operator acts as follows

$$
\bar{\partial}_{\mathrm{L}}: \mathcal{C}^{\infty}\left(\Sigma, L^{k} \otimes K^{s}\right) \rightarrow \Omega^{0,1}\left(\Sigma, L^{k} \otimes K^{s}\right)
$$

from $\mathcal{C}^{\infty}$ sections of $L^{k} \otimes K^{s}$ to $(0,1)$ forms with coefficients in $L^{k} \otimes K^{s}$. For background on the $\bar{\partial}$-equation and holomorphic sections we refer to Ref. [51]. Solutions of Eq. (23) form a vector space $H^{0}\left(\Sigma, L^{k} \otimes K^{s}\right)$. The holomorphic Euler characteristic of $L^{k} \otimes K^{s}$ is calculated by the Riemann-Roch theorem [38, p. 245-6], [51, Theorem 1.4.6],

$$
\chi\left(L^{k} \otimes K^{s}\right):=\operatorname{dim} H^{0}\left(\Sigma, L^{k} \otimes K^{s}\right)-\operatorname{dim} H^{1}\left(\Sigma, L^{k} \otimes K^{s}\right)=\operatorname{deg}\left(L^{k} \otimes K^{s}\right)+1-\mathrm{g} .
$$

Recall that $\operatorname{deg} K=2(\mathrm{~g}-1)$ and by our assumption (20), $\operatorname{deg} L=\int_{\Sigma} c_{1}(L)=1$. Note that a holomorphic line bundle $L$ is positive on $\Sigma$ if and only if $\operatorname{deg} L>0$, cf. [38, p. 214]. On the other hand, by the Kodaira vanishing theorem, $H^{1}\left(\Sigma, L^{k} \otimes K^{s}\right)=0$ if $\operatorname{deg} L^{k} \otimes K^{s}>\operatorname{deg} K$, see [38, p. 215], equivalently, if $k+2(\mathrm{~g}-1)(s-1)>0$. Hence for $s \in \frac{1}{2} \mathbb{Z}$ and $k>2(1-\mathrm{g})(s-1)$ we have

$$
N_{k}=\operatorname{dim} H^{0}\left(\Sigma, L^{k} \otimes K^{s}\right)=k+(1-\mathrm{g})(1-2 s) .
$$

In this paper we assume that the magnetic field flux $k \gg 1$, so that the number of states is always positive and large.

In the symmetric gauge (19), (21), the LLL wave functions solve a closely related equation

$$
\bar{D} \psi_{j}=0,
$$

where the locally-defined operator $\bar{D}$ is nothing but the global operator $\bar{\partial}_{\mathrm{L}}$ in (23), written in the chosen gauge. Namely

$$
\bar{D}=h^{\frac{k}{2}} g_{z \bar{z}}^{-\frac{s+1}{2}} i \bar{\partial}_{L}\left(h^{-\frac{k}{2}} g_{z \bar{z}}^{\frac{s}{2}} \cdot\right)=g_{z \bar{z}}^{-\frac{1}{2}}\left(i \partial_{\bar{z}}-s \omega_{\bar{z}}+A_{\bar{z}}\right)
$$

The half-integer $s$ is called the spin. Consequently, Eq. (26) can be formally solved by twisting the basis in $H^{0}\left(\Sigma, L^{k} \otimes K^{s}\right)$ as follows

$$
\psi_{j}(z, \bar{z})=s_{j}(z) h^{\frac{k}{2}} g_{z \bar{z}}^{-\frac{s}{2}},
$$


where $s_{j}(z)$ is a holomorphic section of $L^{k} \otimes K^{s}$, so the number of solutions of (27) is the same as in Eq. (25).

Norm squared of a section at a point $z \in \Sigma$ is defined with the help of the Hermitian metric, and is given by

$$
\|s(z)\|^{2}=|s(z)|^{2} h^{k}(z, \bar{z}) g_{z \bar{z}}^{-s}(z, \bar{z})
$$

Now $\|s(z)\|^{2}$ is a scalar function on $\Sigma$, which can be integrated. More generally, the $L^{2}$ inner product of sections reads

$$
\left\langle s_{1}, s_{2}\right\rangle=\frac{1}{2 \pi} \int_{\Sigma} \bar{s}_{1} s_{2} h^{k} g_{z \bar{z}}^{-s} \sqrt{g} d^{2} z .
$$

This immediately translates into the standard quantum-mechanical inner product for the wave functions (28),

$$
\left\langle\psi_{1} \mid \psi_{2}\right\rangle=\frac{1}{2 \pi} \int_{\Sigma} \psi_{1}^{*} \psi_{2} \sqrt{g} d^{2} z
$$

Now we are ready to define the generating functional in the integer Quantum Hall.

2.2. Generating functional. Consider a non-normalized basis $\left\{s_{j}\right\}$ of $H^{0}\left(\Sigma, L^{k} \otimes K^{s}\right)$ (later it will be important that the sections $s_{j}$ depend on $Y$ holomorphically) and the corresponding basis of wave functions (28). Then there exists a section of the determinant line bundle $\left(L^{k} \otimes\right.$ $\left.K^{s}\right)_{z_{1}} \wedge \cdots \wedge\left(L^{k} \otimes K^{s}\right)_{z_{N_{k}}}$, associated to the basis $s_{j}$. (More precisely, the line bundle is $\operatorname{det}\left(\oplus_{j} \pi_{j}^{*}\left(L^{k} \otimes K^{s}\right)\right)$ on $\Sigma^{N_{k}}$, where $\pi_{j}$ is the projection on the $j$-th factor of $\Sigma^{N_{k}}$.) This section is given by the completely antisymmetric combination $s_{1} \wedge \ldots \wedge s_{N_{k}}$ and can be formally written as a Slater determinant

$$
\frac{1}{N_{k} !} \operatorname{det}\left[s_{j}\left(z_{l}\right)\right]_{j, l=1}^{N_{k}} \text {. }
$$

This is a collective wave function of free electrons on LLL, which we refer to as the integer QH-state. Physically it means that the lowest Landau level is completely filled, i.e., we have $N_{k}$ particles (fermions) at the positions $z_{1}, \ldots, z_{N_{k}}$. The Hermitian norm of the section (31) is induced by the point-wise norm (29) for each $s_{j}$ and reads

$$
\left|\Psi\left(z_{1}, \ldots, z_{N_{k}}\right)\right|^{2}:=\frac{1}{N_{k} !}\left|\operatorname{det} s_{j}\left(z_{l}\right)\right|^{2} \prod_{j=1}^{N_{k}} h^{k}\left(z_{j}, \bar{z}_{j}\right) g_{z \bar{z}}^{-s}\left(z_{j}, \bar{z}_{j}\right) .
$$

This is a scalar function on $\Sigma^{N_{k}}$, which defines a measure on configuration of $N_{k}$ points (3). The partition function (4) is

$$
Z_{k}=\frac{1}{(2 \pi)^{N_{k}} N_{k} !} \int_{\Sigma^{N_{k}}}\left|\operatorname{det} s_{j}\left(z_{l}\right)\right|^{2} \prod_{j=1}^{N_{k}} h^{k}\left(z_{j}, \bar{z}_{j}\right) g_{z \bar{z}}^{-s}\left(z_{j}, \bar{z}_{j}\right) \sqrt{g} d^{2} z_{j},
$$

and $\log Z_{k}$ is called the generating functional.

Another useful representation of the partition function is in terms of the determinant of the Gram matrix of inner products (30) of sections

$$
Z_{k}=\operatorname{det} \frac{1}{2 \pi} \int_{\Sigma} \bar{s}_{j}(\bar{z}) s_{l}(z) h^{k} g_{z \bar{z}}^{-s} \sqrt{g} d^{2} z=\operatorname{det}\left\langle s_{j}, s_{l}\right\rangle
$$

This representation is called the determinantal formula. From this representation it follows that the integral (32) converges, since the sections are $L^{2}$-integrable (30). 
By construction $Z_{k}$ depends on the choice of the basis in $H^{0}\left(\Sigma, L^{k} \otimes K^{s}\right)$. However, this dependence is not hard to take care of. Note that under the linear change of the basis of sections $s_{i} \rightarrow s_{i}^{\prime}=A_{i j} s_{j}$ the generating functional transforms as

$$
\begin{aligned}
& s_{i} \rightarrow s_{i}^{\prime}=A_{i j} s_{j}, \quad A \in G L\left(N_{k}, \mathbb{C}\right) \\
& Z_{k} \rightarrow \operatorname{det}\left(A^{*} A\right) \cdot Z_{k} .
\end{aligned}
$$

Since $A_{i j}$ is a numerical matrix, the derivatives of $\log Z_{k}$ are $A$-independent, hence they are independent of the choice of the basis of sections. Consider now some reference metrics $h_{0}^{k}$ on $L^{k}$ and $g_{0}$ on $\Sigma$, connected with $h^{k}$ and $g$ as

$$
\begin{aligned}
& h^{k}(z, \bar{z})=h_{0}^{k}(z, \bar{z}) e^{-k \psi(z, \bar{z})} \\
& g_{z \bar{z}}=g_{0 z \bar{z}}+\partial_{z} \partial_{\bar{z}} \phi(z, \bar{z}),
\end{aligned}
$$

where the $\mathcal{C}^{\infty}$ scalar functions $\psi(z, \bar{z})$ and $\phi(z, \bar{z})$ are known as the Kähler potential and the magnetic potential, respectively. In particular, Eq. (35) translates into

$$
F_{z \bar{z}}=F_{0 z \bar{z}}+k \partial_{z} \partial_{\bar{z}} \psi,
$$

Since the metric and the magnetic field are positive, $g_{z \bar{z}}>0, F>0$, the functions $\psi(z, \bar{z})$ and $\phi(z, \bar{z})$ satisfy $\partial_{z} \partial_{\bar{z}} \phi(z, \bar{z})>-g_{0 z \bar{z}}$ and $\partial_{z} \partial_{\bar{z}} \psi>-F_{0 z \bar{z}} / k$.

Consider now the generating functional $\log Z_{k 0}$, defined using the reference metrics. It follows from the transformation rule, Eq. (34), that the difference

$$
\log Z_{k}-\log Z_{k 0}
$$

is independent of the choice of the basis in $H^{0}\left(\Sigma, L^{k} \otimes K^{s}\right)$.

Finally, let us explain why $\log Z_{k}$ is called the generating functional. Writing $h^{k}=h_{0}^{k} e^{-k \psi}$, it is straightforward to see that the variational derivatives of (4) with respect to $\psi$

$$
\frac{\delta}{\delta \psi\left(w_{1}, \bar{w}_{1}\right)} \cdots \frac{\delta}{\delta \psi\left(w_{m}, \bar{w}_{m}\right)} \log Z_{k}=(-k)^{m}\left\langle\rho\left(w_{1}, \bar{w}_{1}\right) \ldots \rho\left(w_{m}, \bar{w}_{m}\right)\right\rangle_{c}
$$

give the connected multi-point correlation functions of electronic density operator $\rho(z, \bar{z})=$ $\sum_{j=1}^{N_{k}} \delta\left(z, z_{j}\right)$. These density correlation functions were considered in [72, 25, 26].

2.3. Determinant line bundle and Quillen metric. In [56] Quillen defined the metric on determinant line bundle, using regularized determinants of the Cauchy-Riemann operators on Riemann surfaces. It was used for the computation of holomorphic anomalies in string theory amplitudes by Belavin-Knizhnik [8]. More generally, the curvature formulas for were derived for determinant lines of Dirac operators by Bismut-Freed [16, Theorem 1.21] and for Kähler manifolds by Bismut-Gillet-Soule [18, Theorem 1.9]. For the relevant literature see also the following physics [2, 67, 30] and mathematical papers [22, 74]. In the context of the integer quantum Hall effect on Riemann surfaces the Quillen metric was first discussed in Refs. [6, 49], see also [43] for the fractional case.

In Appendix A we give a general overview of the Quillen metric and analytic torsion, and here we consider the Riemann surface case, which is of main relevance to this paper.

Consider again the $\bar{\partial}_{L}$ operator (23), which acts from the vector space of sections to the vector space of $(0,1)$-forms (24) . The adjoint operator $\bar{\partial}_{L}^{*}$ relative to the inner product (30) acts in the opposite direction. Hence the (Kodaira) Laplacian can be defined as

$$
\Delta_{\mathrm{L}}=\bar{\partial}_{L}^{*} \bar{\partial}_{L}: \mathcal{C}^{\infty}\left(\Sigma, L^{k} \otimes K^{s}\right) \rightarrow \mathcal{C}^{\infty}\left(\Sigma, L^{k} \otimes K^{s}\right) .
$$


It will be convenient to work with its Hermitian-equivalent. Namely, we consider the operator $\bar{D}$ as defined in (27). The adjoint operator $\bar{D}^{*}$ reads

$$
\bar{D}^{*}=g_{z \bar{z}}^{-\frac{1}{2}}\left(i \partial_{z}-(s-1) \omega_{z}+A_{z}\right) .
$$

Hence we define the Laplacian $\Delta_{s}^{-}$as

$$
\Delta_{s}^{-}=\bar{D}^{*} \bar{D}, \quad \Delta_{s}^{-}: \mathcal{C}^{\infty}\left(\Sigma, L^{k} \otimes K^{s}\right) \rightarrow \mathcal{C}^{\infty}\left(\Sigma, L^{k} \otimes K^{s}\right) .
$$

We will also need to define the Laplacian with the operators above interchanged

$$
\Delta_{s}^{+}=\bar{D} \bar{D}^{*}, \quad \Delta_{s}^{+}: \mathcal{C}^{\infty}\left(\Sigma, L^{k} \otimes K^{s}\right) \rightarrow \mathcal{C}^{\infty}\left(\Sigma, L^{k} \otimes K^{s}\right) .
$$

In particular, setting $s=k=0$ both Laplacians reduce to the usual scalar Laplacian. Since $\operatorname{ker} \bar{D}^{*}=0$, it follows from Riemann-Roch theorem (25) that in our case

$$
\operatorname{dim} \operatorname{ker} \Delta_{s}^{-}=\operatorname{dim} \operatorname{ker} \bar{D}=k+(1-\mathrm{g})(1-2 s) .
$$

The definition the regularized spectral determinants is standard. Given non-zero eigenvalues $\lambda$ of $\Delta_{\mathrm{L}}$, the regularized spectral determinant of the Laplacian is defined as $\operatorname{det}^{\prime} \Delta_{\mathrm{L}}=$ $\exp \left(-\zeta^{\prime}(0)\right)$, where the zeta function is $\zeta(u)=\sum_{\lambda} \lambda^{-u}$. In view of (27), the spectral determinants of both Laplacians (40) and (41) are equal, $\operatorname{det}^{\prime} \Delta_{s}^{-}=\operatorname{det}^{\prime} \Delta_{\mathrm{L}}$. Now we would like to study the dependence of the QH-state (31) and the partition function $Z_{k}$ on the parameter space $Y=\mathcal{M}_{\mathrm{g}}(\Sigma) \times \operatorname{Jac}(\Sigma)(5)$. Recall, that $\mathcal{M}_{\mathrm{g}}(\Sigma)$ is the moduli space of complex structures on $\Sigma$ and $\operatorname{Jac}(\Sigma)$ is the moduli space of flat connections $d A=0$ on $\Sigma$ (Jacobian variety). Given the canonical basis of one-cycles $\left(A_{a}, B_{b}\right) \in H_{1}(\Sigma, \mathbb{Z}), a, b=1, \ldots$, g in $\Sigma$ and the dual basis of harmonic one-forms $\alpha_{a}, \beta_{b} \in H^{1}(\Sigma, \mathbb{Z})$, we can parameterize the flat connections as follows,

$$
A^{\text {flat }}=2 \pi \sum_{a=1}^{\mathrm{g}}\left(\varphi_{1}^{a} \alpha_{a}-\varphi_{2}^{a} \beta_{a}\right) .
$$

Here $\left(\varphi_{1}^{a}, \varphi_{2}^{b}\right) \in[0,1]^{2 \mathrm{~g}}$ are coordinates on the Jacobian, which is $2 \mathrm{~g}$-dimensional torus for the surfaces of genus g. This space corresponds to Aharonov-Bohm solenoid fluxes piercing the handles of the surface.

The Slater determinant (31) is a section $\mathcal{S}$ of the determinant line bundle $\mathcal{L}$ over the parameter space, $\mathcal{L}=\operatorname{det} H^{0}\left(\Sigma, L^{k} \otimes K^{s}\right)$. For the basis $\left\{s_{j}\right\}$ of $H^{0}\left(\Sigma, L^{k} \otimes K^{s}\right)(28)$, the Quillen metric of $\mathcal{S}$ of $\mathcal{L}$ is given by

$$
\|\mathcal{S}\|^{2}=\frac{\operatorname{det}\left\langle s_{j}, s_{l}\right\rangle}{\operatorname{det}^{\prime} \Delta_{\mathrm{L}}}=\frac{Z_{k}}{\operatorname{det}^{\prime} \Delta_{\mathrm{L}}}
$$

where the last equality follows from determinant formula (33).

Example: The metric of the round sphere reads $g_{0 z \bar{z}}=\frac{1}{\left(1+|z|^{2}\right)^{2}}$, where the complex coordinate $z \in \mathbb{C}$, and the constant magnetic field $B=k$ corresponds to the hermitian metric on $L^{k}=\mathcal{O}(k)$

$$
h_{0}^{k}(z)=\frac{1}{\left(1+|z|^{2}\right)^{k}} .
$$

We can choose a basis of holomorphic sections in the form $s_{j}(z)=c_{j} z^{j-1}(d z)^{s}, j=1, \ldots, k-$ $2 s+1$, where $c_{j}$ is a normalization constant. The partition function then reads

$$
Z_{k}^{S^{2}}=\frac{1}{N_{k} !} \prod_{j} \frac{c_{j}^{2}}{2 \pi} \int_{\mathbb{C}^{N_{k}}} \prod_{j<l}\left|z_{j}-z_{l}\right|^{2} \cdot e^{-k \sum_{j} \psi\left(z_{j}\right)-s \sum_{j} \log \frac{\sqrt{g}}{\sqrt{g 0}} \mid z_{j}} \prod_{j=1}^{k-2 s+1} \frac{d^{2} z_{j}}{\left(1+\left|z_{j}\right|^{2}\right)^{k-2 s+2}},
$$


where we used the formula det $z_{l}^{j-1}=\prod_{j<l}\left(z_{j}-z_{l}\right)$ for the Vandermonde determinant. On the sphere there is no complex structure moduli and $H^{1}\left(S^{2}, \mathbb{Z}\right)$ is trivial, so the partition function is only a functional of the potential functions $\phi, \psi$.

Example: On the flat torus $T^{2}=\mathbb{C} / \Lambda, \Lambda=m+n \tau, m, n \in \mathbb{Z}$ the metric reads $g_{0 z \bar{z}}=\frac{2 \pi i}{\tau-\bar{\tau}}$. We define a holomorphic line bundle $L \rightarrow T^{2}$ as follows. Let $\Phi$ be the action of $\Lambda$ on $\mathbb{C} \times \mathbb{C}$ given by $\Phi(1)(z, \xi)=(z+1, \xi), \Phi(\tau)(z, \xi)=\left(z+\tau, e^{-2 \pi i z-\pi i \tau} \xi\right)$. We define $L=\mathbb{C} \times \mathbb{C} / \Lambda$, the quotient space by this action, which is a holomorphic line bundle on $\mathbb{C} / \Lambda$ of degree one. Let $L_{\varphi}=\mathbb{C} \times \mathbb{C} / \Lambda$ with the $\Lambda$ action given by $\Phi_{\varphi}(1)(z, \xi)=(z+1, \xi), \Phi_{\varphi}(\tau)(z, \xi)=\left(z+\tau, e^{-2 \pi i \varphi} \xi\right)$. Constant magnetic field corresponds to the hermitian metric on $L^{k} \otimes L_{\varphi}$,

$$
h_{0}^{k}(z, \bar{z} \mid \varphi, \bar{\varphi})=e^{\frac{\pi i k}{\tau-\bar{\tau}}(z-\bar{z})^{2}+\frac{2 \pi i}{\tau-\bar{\tau}}(z-\bar{z})(\varphi-\bar{\varphi})}, \quad z \in \mathbb{C} .
$$

In this case we have a moduli parameter $\tau \in \mathbb{H} / P S L(2, \mathbb{Z})$ and also Jacobian variety comes into play. We have two 1-cycles and correspondingly two dual harmonic one-forms $\alpha^{1}, \beta^{1}$, and the gauge connection (42) has the form

$$
A^{\text {flat }}=2 \pi\left(\varphi_{1} \alpha^{1}-\varphi_{2} \beta^{1}\right)
$$

The complex coordinate on the Jacobian is defined as $\varphi=\varphi_{2}+\varphi_{1} \tau$. The basis of sections can be written in terms of the theta functions with characteristics. Also the canonical bundle on the torus is trivial, so we can set $s=0$. Using standard notations for the theta functions 53 ] we can choose the basis of $H^{0}\left(\mathbb{C} / \Lambda, L^{k} \otimes L_{\varphi}\right)$ as

$$
s_{j}(z)=\vartheta_{\frac{j}{k}, 0}(k z+\varphi, k \tau), j=1, \ldots k .
$$

Using the $L^{2}$ norm of the section

$$
\frac{1}{2 \pi} \int_{T^{2}} \bar{s}_{j}(\bar{z}) s_{l}(z) h_{0}^{k} \sqrt{g_{0}} d^{2} z=\sqrt{\frac{i}{k(\tau-\bar{\tau})}} \cdot e^{-\frac{\pi i}{k} \frac{(\varphi-\bar{\varphi})^{2}}{\tau-\bar{\tau}}} \delta_{j l},
$$

we can find the partition function $Z_{k}$ for the flat torus and constant magnetic field

$$
Z_{k}^{T^{2}}(\tau, \bar{\tau}, \varphi, \bar{\varphi})=\frac{1}{k^{k / 2}(2 \operatorname{Im} \tau)^{k / 2}} \cdot e^{-\pi i \frac{(\varphi-\bar{\varphi})^{2}}{\tau-\bar{\tau}}} .
$$

In this form the dependence of the partition function on the parameter space coordinate $y=$ $(\tau, \varphi)$ is explicit (recall that $Z_{k}$ is defined up to multiplication by mod squared of non-vanishing holomorphic function of $\tau$ ).

\section{LARGe $k$ ASYMPtotics OF THE GENERATING FUNCTIONAL}

3.1. Proof of Theorem 1. The following is a more detailed formulation of the Theorem 1. Here we write the formula Eq. (51) for the generation functional in terms of the Kähler and magnetic potentials. This formulation is equivalent to Eq. (11) up to integration by parts.

Theorem 1. Consider the difference of generating functionals $\log Z_{k}-\log Z_{k 0}$ (38) for the metrics $h_{0}^{k}, h^{k}$ on $L^{k}$ and $g_{0}, g$ on $\Sigma$, as defined in Eqs. (35, 36). The following asymptotic expansion holds at large $k$,

$$
\log \frac{Z_{k}}{Z_{k 0}}=\log \frac{Z_{H}}{Z_{H 0}}+\mathcal{F}-\mathcal{F}_{0}
$$


where the non-local (anomalous) part $\log Z_{H}$ is given by (11). The difference $\log Z_{H}-\log Z_{H 0}$ consists of three leading orders in $k$

$$
\log \frac{Z_{H}}{Z_{H 0}}=-k^{2} S_{2}(\psi)+k \frac{(1-2 s)}{2} S_{1}(\phi, \psi)+\left(\frac{(1-2 s)^{2}}{4}-\frac{1}{12}\right) S_{L}(\phi) .
$$

where $S_{2}, S_{1}, S_{L}$ are geometric functionals. They read

$$
\begin{aligned}
& S_{2}(\psi)=\frac{1}{2 \pi} \int_{\Sigma}\left(\frac{1}{4} \psi \Delta_{0} \psi+\frac{1}{k} B_{0} \psi\right) \sqrt{g_{0}} d^{2} z \\
& S_{1}(\phi, \psi)=\frac{1}{2 \pi} \int_{\Sigma}\left(-\frac{1}{2} \psi R_{0}+\left(\frac{1}{k} B_{0}+\frac{1}{2} \Delta_{0} \psi\right) \log \left(1+\frac{1}{2} \Delta_{0} \phi\right)\right) \sqrt{g_{0}} d^{2} z \\
& S_{L}(\phi)=\frac{1}{2 \pi} \int_{\Sigma}\left(-\frac{1}{4} \log \left(1+\frac{1}{2} \Delta_{0} \phi\right) \Delta_{0} \log \left(1+\frac{1}{2} \Delta_{0} \phi\right)+\frac{1}{2} R_{0} \log \left(1+\frac{1}{2} \Delta_{0} \phi\right)\right) \sqrt{g_{0}} d^{2} z
\end{aligned}
$$

Here $\Delta_{0}$ is the scalar Laplacian in the reference metric $g_{0}$ and $B_{0}=g_{0}^{z \bar{z}} F_{0 z \bar{z}}$ is the reference magnetic field.

The first terms of the expansion of the exact part $\mathcal{F}$ in (50) are given by

$$
\mathcal{F}=-\frac{1}{2 \pi} \int_{\Sigma}\left[\frac{1}{2} B \log \frac{B}{2 \pi}+\frac{2-3 s}{12} R \log \frac{B}{2 \pi}+\frac{1}{24}(\log B) \Delta_{g}(\log B)\right] \sqrt{g} d^{2} z+\mathcal{O}(1 / k) .
$$

Proof. We begin with the variational formula for the generating functional $Z_{k}$, starting from the determinant formula (33). Denoting $G_{j l}=\left\langle s_{j}, s_{l}\right\rangle$, we get

$$
\begin{aligned}
\delta \log Z_{k} & =\delta \operatorname{Tr} \log \left\langle s_{j}, s_{l}\right\rangle=-\frac{1}{2 \pi} \sum_{j, l} G_{l j}^{-1} \int_{\Sigma}\left(\frac{s-1}{2}\left(\Delta_{g} \delta \phi\right)+k \delta \psi\right) \bar{s}_{j} s_{l} h^{k} g_{z \bar{z}}^{-s} \sqrt{g} d^{2} z \\
& =-\frac{1}{2 \pi} \int_{\Sigma}\left(\frac{s-1}{2}\left(\Delta_{g} B_{k}(z, \bar{z})\right) \delta \phi+k B_{k}(z, \bar{z}) \delta \psi\right) \sqrt{g} d^{2} z
\end{aligned}
$$

where $B_{k}(z, \bar{z})$ is the Bergman kernel on the diagonal for the line bundle $L^{k} \otimes K^{s}$. Once we know the large $k$ asymptotic expansion of the Bergman kernel on the diagonal we can determine the asymptotic expansion of the generating functional. The asymptotic expansion of the Bergman kernel is reviewed in the Appendix B. There it is shown (see Eq. (135)) that as $k \rightarrow \infty$,

$$
B_{k}(z, \bar{z})=B+\frac{1-2 s}{4} R+\frac{1}{4} \Delta_{g} \log B+\frac{2-3 s}{24} \Delta_{g}\left(B^{-1} R\right)+\frac{1}{24} \Delta_{g}\left(B^{-1} \Delta_{g} \log B\right)+\mathcal{O}\left(1 / k^{2}\right) .
$$

Let us comment on the orders of different terms in this expansion. The magnetic field $B$ is assumed to be of order $B \sim k \gg 1$, meaning $B(z, \bar{z}) / k$ is a smooth positive function on $\Sigma$, independent of $k$. Therefore the first term here is of order $k$, the second and third terms have order one and the last two terms have order $1 / k$. The inverse of $B$ is well-defined due to the positivity condition $B>0$. Note, that the expansion above is a gradient expansion, in terms of derivatives of the Riemannian metric and magnetic field. 
Next we substitute the asymptotic expansion (57) to the variational formula (56) and rearrange the terms as follows

$$
\begin{aligned}
\delta \log Z_{k}= & -\frac{1}{2 \pi} \int_{\Sigma} k B \delta \psi \sqrt{g} d^{2} z+k \frac{1-2 s}{8 \pi} \int_{\Sigma}\left(-R \delta \psi+\frac{1}{k}\left(\Delta_{g} B\right) \delta \phi\right) \sqrt{g} d^{2} z \\
& -\frac{1}{8 \pi} \int_{\Sigma}\left(k\left(\Delta_{g} \log B\right) \delta \psi-\left(\Delta_{g} B\right) \delta \phi\right) \sqrt{g} d^{2} z \\
& +\left(\frac{(1-2 s)^{2}}{4}-\frac{1}{12}\right) \frac{1}{8 \pi} \int_{\Sigma}\left(\Delta_{g} R\right) \delta \phi \sqrt{g} d^{2} z \\
& -\frac{2-3 s}{48 \pi} \int_{\Sigma}\left(k \Delta_{g}\left(B^{-1} R\right) \delta \psi-\left(\Delta_{g}^{2} \log B\right) \delta \phi-\left(\Delta_{g} R\right) \delta \phi\right) \sqrt{g} d^{2} z \\
& -\frac{1}{48 \pi} \int_{\Sigma}\left(k \Delta_{g}\left(B^{-1} \Delta_{g} \log B\right) \delta \psi-\left(\Delta_{g}^{2} \log B\right) \delta \phi\right) \sqrt{g} d^{2} z .
\end{aligned}
$$

Writing out the definitions (22), (37) explicitly,

$$
B=g^{z \bar{z}} F_{z \bar{z}}=\frac{B_{0}+\frac{1}{2} k \Delta_{0} \psi}{1+\frac{1}{2} \Delta_{0} \phi}, \quad R=-\Delta_{g} \log \sqrt{g}=\frac{R_{0}-\Delta_{0} \log \left(1+\frac{1}{2} \Delta_{0} \phi\right)}{1+\frac{1}{2} \Delta_{0} \phi},
$$

(note that $B_{0} / k$ is independent of $k$ ) one can directly verify the following variational formulas for the geometric functionals (52)-(54),

$$
\begin{aligned}
\delta S_{2} & =\frac{1}{2 \pi} \int_{\Sigma} \frac{1}{k} B \delta \psi \sqrt{g} d^{2} z \\
\delta S_{1} & =\frac{1}{4 \pi} \int_{\Sigma}\left(-R \delta \psi+\frac{1}{k}\left(\Delta_{g} B\right) \delta \phi\right) \sqrt{g} d^{2} z, \\
\delta S_{L} & =\frac{1}{8 \pi} \int_{\Sigma}\left(\Delta_{g} R\right) \delta \phi \sqrt{g} d^{2} z
\end{aligned}
$$

and also

$$
\begin{aligned}
& \delta \int_{\Sigma} B \log B \sqrt{g} d^{2} z=\frac{1}{2} \int_{\Sigma}\left(k\left(\Delta_{g} \log B\right) \delta \psi-\left(\Delta_{g} B\right) \delta \phi\right) \sqrt{g} d^{2} z \\
& \delta \int_{\Sigma} R \log B \sqrt{g} d^{2} z=\frac{1}{2} \int_{\Sigma}\left(k\left(\Delta_{g}\left(B^{-1} R\right)\right) \delta \psi-\left(\Delta_{g}^{2} \log B\right) \delta \phi-\left(\Delta_{g} R\right) \delta \phi\right) \sqrt{g} d^{2} z, \\
& \delta \int_{\Sigma}(\log B) \Delta_{g}(\log B) \sqrt{g} d^{2} z=\int_{\Sigma}\left(k\left(\Delta_{g}\left(B^{-1} \Delta_{g} \log B\right)\right) \delta \psi-\left(\Delta_{g}^{2} \log B\right) \delta \phi\right) \sqrt{g} d^{2} z .
\end{aligned}
$$

Now we can integrate Eq. (58) term by term. In the first line of (58) we recognize the variations of the functionals $S_{2}$ and $S_{1}$, in the second line - the variation of (62), in the third line - the variation of $S_{L}$ (54), and the variations of (63), (64) in the last two lines of (58).

When integrating this formulas, we need to take into account the obvious boundary condition: at $\phi=\psi=0$ we have $\log Z_{k}-\log Z_{k 0}=0$. This completes the proof.

Remarks: Using Theorem 2, we interpret $\mathcal{F}$ as $\log \operatorname{det}^{\prime} \Delta_{\mathrm{L}}$. While we computed $\mathcal{F}$ to the order $\mathcal{O}(1)$, in fact that all $1 / k$-corrections to $\log \operatorname{det}^{\prime} \Delta_{\mathrm{L}}$ are given by local functionals. This follows from the general arguments given in [51, §5.5.4]. We also note that physics proof of the locality of $\mathcal{F}$ was also given in Ref. [34, §5]. 
Integrating the left hand side of (57) over the surface, we should get the total number of LLL states. We have

$$
N_{k}=\frac{1}{2 \pi} \int_{\Sigma} B_{k}(z, \bar{z}) \sqrt{g} d^{2} z=k+(1-\mathrm{g})(1-2 s),
$$

in accordance with (25). Indeed, only the first two terms on the right hand side in (57) contribute to the integral, while the rest of the expansion is a total derivative. Let us also mention that the Bergman kernel is the average density of states

$$
\langle\rho(z, \bar{z})\rangle=\frac{1}{2 \pi} B_{k}(z, \bar{z})
$$

as follows from (56) and (39).

In the case of constant magnetic field $B=k$ and $s=0$ the expansion (51) is in agreement2 with the one obtained in [42. In particular, functionals $S_{1}$ (52) and $S_{2}$ (53) reduce to the combinations of the Aubin-Yau and Mabuchi functionals in Kähler geometry. The functional $S_{L}$ (54) is the Liouville functional. The exact terms in Eq. (55) are in agreement with [26, Eq. $(130)]$.

As we have mentioned, the formula Eq. (11) for the anomalous part $\log Z_{H}$ of the generating functional

$$
\begin{aligned}
\log \frac{Z_{H}}{Z_{H 0}}= & \frac{2}{\pi} \int_{\Sigma}\left[A_{z} A_{\bar{z}}+\frac{1-2 s}{2}\left(A_{z} \omega_{\bar{z}}+\omega_{z} A_{\bar{z}}\right)+\left(\frac{(1-2 s)^{2}}{4}-\frac{1}{12}\right) \omega_{z} \omega_{\bar{z}}\right] d^{2} z \\
& -\left(A \rightarrow A_{0}, \omega \rightarrow \omega_{0}\right)
\end{aligned}
$$

follows from (51) by integration by parts. This formula is understood to be valid specifically in the symmetric gauge, where the coefficients of the gauge connection and spin connection 1-forms (19), (21). This is a somewhat formal, but nevertheless useful way to represent Eq. (11), because it resembles Chern-Simons functional, which will appear in \$5.1.

We emphasize that the anomalous part is a non-local functional of magnetic field and the curvature, while the exact terms are local, i.e., involve integrals of the magnetic field density and scalar curvature and their derivatives at a point. This can be illustrated by another non-local representation for the anomalous term via double integrals

$$
\begin{aligned}
\log \frac{Z_{H}}{Z_{H 0}}= & -\left.\left.\frac{1}{2 \pi} \int_{\Sigma \times \Sigma}\left(B+\frac{1-2 s}{4} R\right)\right|_{z} \Delta_{g}^{-1}\left(z, z^{\prime}\right)\left(B+\frac{1-2 s}{4} R\right)\right|_{z^{\prime}} \sqrt{g} d^{2} z \sqrt{g} d^{2} z^{\prime} \\
& +\left.\left.\frac{1}{96 \pi} \int_{\Sigma \times \Sigma} R\right|_{z} \Delta_{g}^{-1}\left(z, z^{\prime}\right) R\right|_{z^{\prime}} \sqrt{g} d^{2} z \sqrt{g} d^{2} z^{\prime}-\left(R \rightarrow R_{0}, B \rightarrow B_{0}\right),
\end{aligned}
$$

where $\Delta_{g}^{-1}$ is the inverse Laplace operator. This formula is an analog of the Polyakov effective action in $2 d$ gravity [55], in the presence of the magnetic field $B$.

\footnotetext{
${ }^{2}$ The Riemannian conventions for the scalar curvature and Laplacian used here are different by a factor 2 from Kähler conventions used in 42. Then the expansion of the Bergman kernel in 42 follows from (57) after setting the magnetic field to constant: $B=k$.
} 


\section{Regularized SPECTRAL DEterminant of the LAPLACiAN}

In $\$ 2.3$ we have seen that the norm squared $\|\mathcal{S}\|^{2}$ of a section of the determinant line bundle $\mathcal{L}$ on $Y$ is given by the ratio of the generating functional $Z_{k}$ and regularized determinant of the Laplacian for the line bundle (43). Here we show that the anomalous part of the generating functional (51) is encoded in the Quillen metric (43). The following is a more detailed formulation of Theorem 2. We will use the small time expansion of the heat kernel taking advantage of the fact that this expansion is easy to handle on Riemann surfaces. Thus we give a direct proof here, avoiding the sophisticated local index arguments from [18, Theorem 1.23].

TheOREM 4. Consider the metrics $h_{0}^{k}, h^{k}$ on $L^{k}$ and $g_{0}, g$ on $\Sigma$ related as in Eqs. (35), (36). The norm of the determinant section $\|\mathcal{S}\|^{2}$ is defined as in Eq. (43) for the metrics $g, h^{k}$, and $\|\mathcal{S}\|_{0}^{2}$ for the metrics $g_{0}, h_{0}^{k}$. Then the following exact transformation formula holds

$$
\log \frac{\|\mathcal{S}\|^{2}}{\|\mathcal{S}\|_{0}^{2}}=-k^{2} S_{2}(\psi)+k \frac{(1-2 s)}{2} S_{1}(\phi, \psi)+\left(\frac{(1-2 s)^{2}}{4}-\frac{1}{12}\right) S_{L}(\phi),
$$

where the functionals are defined in Eqs. (52)-(54).

Proof. The derivation here is based on the standard heat kernel technique, (see e. g. [30, §IIF], where the canonical line bundle $K^{s}$ was considered.) We begin with the heat kernel representation of the regularized determinant

$$
\log \operatorname{det}^{\prime} \Delta_{s}^{-}=-\int_{\epsilon}^{\infty} \frac{d t}{t}\left(\operatorname{Tr} e^{-t \Delta_{s}^{-}}-N_{k}\right),
$$

where $\epsilon \rightarrow 0$. Now we compute the variation with respect to the Kähler potential $\delta \phi$ and magnetic potential $\delta \psi$

$$
\delta \log \operatorname{det}^{\prime} \Delta_{s}^{-}=\int_{\epsilon}^{\infty} d t \operatorname{Tr} \delta \Delta_{s}^{-} e^{-t \Delta_{s}^{-}} .
$$

The variations of the first-order operators are given by

$$
\begin{aligned}
& \delta \bar{D}=-\frac{1}{4}\left[(s+1) \Delta_{g} \delta \phi+2 k \delta \psi\right] \bar{D}+\frac{1}{4} \bar{D}\left[s \Delta_{g} \delta \phi+2 k \delta \psi\right], \\
& \delta \bar{D}^{*}=\frac{1}{4}\left[(s-2) \Delta_{g} \delta \phi+2 k \delta \psi\right] \bar{D}^{*}-\frac{1}{4} \bar{D}^{*}\left[(s-1) \Delta_{g} \delta \phi+2 k \delta \psi\right] .
\end{aligned}
$$

Using the formulas above we derive

$$
\begin{aligned}
\operatorname{Tr} \delta \Delta_{s}^{-} e^{-t \Delta_{s}^{-}}= & \operatorname{Tr}\left(\delta \bar{D}^{*} \bar{D}+\bar{D}^{*} \delta \bar{D}\right) e^{-t \Delta_{s}^{-}} \\
= & \operatorname{Tr}\left(\frac{1}{4}\left[(s-2) \Delta_{g} \delta \phi+2 k \delta \psi\right] \Delta_{s}^{-}-\frac{1}{4} \bar{D}^{*}\left[(s-1) \Delta_{g} \delta \phi+2 k \delta \psi\right] \bar{D}\right. \\
& \left.-\frac{1}{4} \bar{D}^{*}\left[(s+1) \Delta_{g} \delta \phi+2 k \delta \psi\right] \bar{D}+\frac{1}{4} \Delta_{s}^{-}\left[s \Delta_{g} \delta \phi+2 k \delta \psi\right]\right) e^{-t \Delta_{s}^{-}} \\
= & \operatorname{Tr}\left(\frac{s-1}{2} \Delta_{g} \delta \phi+k \delta \psi\right) \Delta_{s}^{-} e^{-t \Delta_{s}^{-}}-\operatorname{Tr}\left(\frac{s}{2} \Delta_{g} \delta \phi+k \delta \psi\right) \Delta_{s-1}^{+} e^{-t \Delta_{s-1}^{+}}
\end{aligned}
$$


Here in the middle terms we used the rearrangement identity $\operatorname{Tr} \bar{D} e^{-t \Delta_{s}^{-}} \bar{D}^{*}=\operatorname{Tr} \Delta_{s-1}^{+} e^{-t \Delta_{s-1}^{+}}$. Going back to (67) and performing $t$-integral we obtain

$$
\delta \log \operatorname{det}^{\prime} \Delta_{s}^{-}=-\left.\operatorname{Tr}\left(\frac{s-1}{2} \Delta_{g} \delta \phi+k \delta \psi\right) e^{-t \Delta_{s}^{-}}\right|_{\epsilon} ^{\infty}+\left.\operatorname{Tr}\left(\frac{s}{2} \Delta_{g} \delta \phi+k \delta \psi\right) e^{-t \Delta_{s-1}^{+}}\right|_{\epsilon} ^{\infty} .
$$

As $t \rightarrow \infty$ the first heat kernel above is projected onto its zero modes (28) and the second one vanishes, since ker $\Delta_{s}^{+}=0$. The $t=\infty$ part reduces to

$$
\begin{aligned}
-\left.\operatorname{Tr}\left(\frac{s-1}{2} \Delta_{g} \delta \phi+k \delta \psi\right) e^{-t \Delta_{s}^{-}}\right|_{t=\infty} & =-\sum_{j=1}^{N_{k}}\left\langle s_{j},\left(\frac{s-1}{2} \Delta_{g} \delta \phi+k \delta \psi\right) s_{j}\right\rangle \\
& =-\delta \log \operatorname{det}\left\langle s_{j}, s_{l}\right\rangle,
\end{aligned}
$$

where we recognize the variation formula (56) for the generating functional in the determinantal representation (33). Using (70), Eq. (69) can be now written as

$$
\delta \log \frac{\operatorname{det} \Delta_{s}^{-}}{Z_{k}}=\left.\operatorname{Tr}\left(\frac{s-1}{2} \Delta_{g} \delta \phi+k \delta \psi\right) e^{-\epsilon \Delta_{s}^{-}}\right|_{\epsilon \rightarrow 0}-\left.\operatorname{Tr}\left(\frac{s}{2} \Delta_{g} \delta \phi+k \delta \psi\right) e^{-\epsilon \Delta_{s-1}^{+}}\right|_{\epsilon \rightarrow 0} .
$$

We evaluate these terms with the help of the short-time expansion formulas for the heat kernel on the diagonal

$$
\begin{aligned}
& \left\langle z\left|e^{-\epsilon \Delta_{s}^{-}}\right| z\right\rangle=\frac{1}{2 \pi \epsilon}+\frac{B}{4 \pi}+\frac{1-3 s}{24 \pi} R+\mathcal{O}(\epsilon), \\
& \left\langle z\left|e^{-\epsilon \Delta_{s-1}^{+}}\right| z\right\rangle=\frac{1}{2 \pi \epsilon}-\frac{B}{4 \pi}+\frac{-2+3 s}{24 \pi} R+\mathcal{O}(\epsilon) .
\end{aligned}
$$

Plugging this in Eq. (71) we note that the singular $1 / \epsilon$ terms cancel and we recognize the variational formulas (59)-(61) for the geometric functionals,

$$
\delta \log \frac{\operatorname{det}^{\prime} \Delta_{s}^{-}}{Z_{k}}=k^{2} \delta S_{2}(\psi)-k \frac{(1-2 s)}{2} \delta S_{1}(\phi, \psi)-\left(\frac{(1-2 s)^{2}}{4}-\frac{1}{12}\right) \delta S_{L}(\phi) .
$$

Integrating this variational formula with the boundary condition $\|\mathcal{S}\|^{2}=\|\mathcal{S}\|_{0}^{2}$ at $\phi=\psi=0$ completes the proof.

As we have already pointed out, this is a special case of a more general anomaly formula for the Quillen metric, [18, Theorem 1.23], which holds for any Kähler manifold, see also [33, Proposition 3.8], see Appendix A for more details. Note also that the formula Eq. (66) is exact and the magnetic field was not assumed to be large in the derivation of this result.

The following corollary follows from Theorems 1 and 2 .

Corollary. The regularized spectral determinant of the Laplacian (41) satisfies the following transformation formula

$$
\log \frac{\operatorname{det}^{\prime} \Delta_{\mathrm{L}}}{\operatorname{det}^{\prime} \Delta_{\mathrm{L} 0}}=\mathcal{F}-\mathcal{F}_{0}
$$

where we used the equality $\operatorname{det}^{\prime} \Delta_{\mathrm{L}}=\operatorname{det}^{\prime} \Delta_{s}^{-}$for the Laplacian in Eq. (41) and $\mathcal{F}$ is given by Eq. (55). 
The first term $\int_{\Sigma} B \log B$ in (55) agrees with the result of Bismut-Vasserot [20] for the leading asymptotics of the analytic torsion for higher powers of line bundles on Kähler manifolds, see also [51, Theorem 5.5.8]. The last two terms in (55) are $\mathcal{O}(1)$ corrections to their result. We conjecture that the coefficient of $k^{0}$ in $\mathcal{F}$ from (56) is the coefficient of $k^{0}$ in the expansion for $\log \operatorname{det}^{\prime} \Delta_{\mathrm{L}}$, which would refine Bismut-Vasserot's result.

Example: On the round sphere with the line bundle $L^{k}=\mathcal{O}(k)$ with the hermitian metric (44), the regularized determinant of Laplacian is given by

$$
\log \operatorname{det}^{\prime} \Delta_{L^{k}}=2 \sum_{j=1}^{k}(k-j) \log (j+1)-(k+1) \log (k+1) !-4 \zeta^{\prime}(-1)+\frac{(k+1)^{2}}{2},
$$

see [45, Theorem 18] 3. By the Euler-Maclaurin formula, at large $k$ the equation above reduces to

$$
\log \operatorname{det}^{\prime} \Delta_{L^{k}}=-\frac{k}{2} \log \frac{k}{2 \pi}-\frac{2}{3} \log k+\mathcal{O}(1)
$$

which is in accordance with Eq. (73) (the scalar curvature of round sphere is $R_{0}=4$ ), where the exact part is given by Eq. (55).

Example: On the flat torus of area $2 \pi$ with the line bundle $L^{k} \otimes L_{\varphi}$ of degree $k>0$, endowed with its canonical translation invariant curvature metric (46), the regularized determinant of Laplacian equals (cf. [21, Prop. 4.2], [12, pp. 4469]),

$$
\log \operatorname{det}^{\prime} \Delta_{L^{k} \otimes L_{\varphi}}=-\frac{k}{2} \log \frac{k}{2 \pi} .
$$

This is actually an exact formula and the reminder term $o(k)$ vanishes. Moreover, the result does not depend on the flat line bundle (47). Combining this with Eq. (49), the norm of the section in the Quillen metric (43) reads

$$
\|\mathcal{S}\|^{2}=\frac{1}{(4 \pi \operatorname{Im} \tau)^{k / 2}} \cdot e^{-\pi i \frac{(\varphi-\bar{\varphi})^{2}}{\tau-\bar{\tau}}}
$$

In particular, this result implies that the adiabatic curvature $\Omega$ and the Chern curvature $\Omega^{\mathcal{L}}$ of the Quillen metric (8) coincide and equal to

$$
\Omega=\Omega^{\mathcal{L}}=\frac{2 \pi i}{\tau-\bar{\tau}} i d \varphi \wedge d \bar{\varphi}-\frac{k}{2(\tau-\bar{\tau})^{2}} i d \tau \wedge d \bar{\tau} .
$$

This is exactly the formula derived in Ref. [7]. The first term here is a $(1,1)$-form on $J a c(\Sigma)$ and describes Hall conductance, as we explained in $\$ 1.1$, and the second term is a $(1,1)$-form on $\mathcal{M}_{1}$ related to Hall viscosity.

Example: On higher genus surfaces the determinant of laplacian for the metric of constant scalar curvature and canonical line bundle has the form, see e.g. [6, Eq. 15] and [29],

$$
\operatorname{det}^{\prime} \Delta_{L^{k}}=e^{-c_{k} \chi(\Sigma)} \prod_{\gamma} \prod_{j=1}^{\infty}\left[1-e^{i \sum_{a=1}^{2 \mathrm{~g}} \varphi_{a} n_{a}(\gamma)} e^{-(j+k) l(\gamma)}\right] .
$$

\footnotetext{
${ }^{3}$ Cf. also [69, §5.1], with the caveat that the metric (44) is used incorrectly and the final result at p. 352 needs to be corrected.
} 
This is written for the surfaces realized as orbit spaces of discrete subgroups $\Gamma$ of $S L(2, \mathbb{R})$ acting on upper half plane. Here $\gamma \in \Gamma$ are primitive hyperbolic elements of $\Gamma$ representing conjugacy classes corresponding to closed geodesics on $\Sigma, n_{a}$ counts the number of times the closed geodesic goes around the ath fundamental loop, and $l(\gamma)$ is the length of geodesic. Also $c_{k}$ is a constant, see [29] for details. The leading term in the asymptotic of $\operatorname{det}^{\prime} \Delta_{L^{k}}$ is given by $e^{-k l\left(\gamma_{\min }\right)}$, where $\gamma_{\text {min }}$ is the length of shortest geodesics. Therefore when the latter is non-vanishing, i.e., away from the boundary of the moduli space this term represents small fluctuations and $\Omega^{\mathcal{L}}$ approximates $\Omega$ with exponential precision.

\section{Curvature formula for Quillen metric and adiabatic transport}

5.1. Curvature formula and adiabatic transport in QHE. In this section we discuss the adiabatic curvature of the integer quantum Hall state and its relation to the Quillen metric. Here we consider the family of Riemann surfaces $\Sigma_{y}$, parameterized by $y \in Y$. Let $M$ be the space, which is the union of all $\Sigma_{y}$ over $Y$ (universal curve), and denote by $\sigma: M \rightarrow Y$ the natural projection. Consider also the family of line bundles $L_{y}^{k} \otimes K_{y}^{s} \rightarrow \Sigma_{y}, y \in Y$. This extends to the holomorphic line bundle $E$ over $M$, which is the union of all line bundles $L_{y}^{k} \otimes K_{y}^{s}$. The Hermitian metric $h^{k}(z, \bar{z}, y, \bar{y})$ straightforwardly extends to the Hermitian metric $h^{E}$ on $E$ over $M$.

Lemma 1. The adiabatic connection equals the Chern connection of the $L^{2}$-metric on $\mathcal{L}$. Hence, the adiabatic curvature $\Omega$ is the curvature of the Chern connection of the $L^{2}$-metric on $\mathcal{L}$.

Proof. As we explained in $\$ 1.1$, in quantum mechanics the adiabatic connection is defined as follows 60. Let $\Psi(t)$ be a normalized wave function $\langle\Psi(t), \Psi(t)\rangle_{L^{2}}=1$, belonging to some (multi-dimensional) parameter space $t \in Y$. Then the adiabatic (Berry) connection is given by the formula Eq. (11),

$$
\mathcal{A}_{t}=i\left\langle\Psi(t), d_{t} \Psi(t)\right\rangle_{L^{2}}
$$

In the setup of the present paper we need a slightly more general version of this connection, due to the following. Our wave function $\mathcal{S}(y)$ Eq. (6) depends on the coordinates $t=(y, \bar{y})$ on the parameter space $Y$ holomorphically and, in fact, it is a section of the line bundle $\mathcal{L}$ on $Y$ as well as $\mathrm{L}$ on $\Sigma$ (for each coordinate $z_{j}$ ). Therefore the derivative $d_{t}$ in the definition above in our context is replaced by the appropriate covariant derivative $\nabla_{u^{H}}^{E}$, which is the Chern connection on $E$, see Eq. (107)-(109). Introducing the notation $\Psi(y, \bar{y})=\mathcal{S}(y) /\|\mathcal{S}(y)\|_{L^{2}}$, where $\Psi(y, \bar{y})$ is the normalized wave function $\langle\Psi(y, \bar{y}), \Psi(y, \bar{y})\rangle_{L^{2}}=1$, we shall define the adiabatic connection $\mathcal{A}_{y}$ in this general setup by

$$
\mathcal{A}_{y}(u)=i\left\langle\Psi(y, \bar{y}), \nabla_{u^{H}}^{E} \Psi(y, \bar{y})\right\rangle_{L^{2}}, \quad \text { for any } u \in T^{(1,0)} Y,
$$

where $u^{H}$ is horizontal lift of $u$. Now we would like to show that

$$
\mathcal{A}_{y}=\frac{i}{2} \partial_{y} \log \|\mathcal{S}(y)\|_{L^{2}}^{2}
$$


We have

$$
\begin{aligned}
& \mathcal{A}_{y}(u)=i\left\langle\mathcal{S}(y) /\|\mathcal{S}(y)\|_{L^{2}}, \nabla_{u^{H}}^{E}\left(\mathcal{S}(y) /\|\mathcal{S}(y)\|_{L^{2}}\right)\right\rangle_{L^{2}} \\
& =i\left\langle\mathcal{S}(y) /\|\mathcal{S}(y)\|_{L^{2}},\left(\nabla_{u^{H}}^{E} \mathcal{S}(y)\right) /\|\mathcal{S}(y)\|_{L^{2}}\right\rangle_{L^{2}}-\frac{i}{2} i_{u} \partial_{y} \log \|\mathcal{S}(y)\|_{L^{2}}^{2} \\
& =i \frac{1}{\|\mathcal{S}(y)\|_{L^{2}}}\left\langle\mathcal{S}(y),\left(\nabla_{u^{H}}^{E} \mathcal{S}(y)\right)\right\rangle_{L^{2}}-\frac{i}{2} i_{u} \partial_{y} \log \|\mathcal{S}(y)\|_{L^{2}}^{2},
\end{aligned}
$$

where $i_{u}$ is contraction with the vector $u$. Then the first term in the last line equals

$$
\left\langle\mathcal{S}(y),\left(\nabla_{u^{H}}^{E} \mathcal{S}(y)\right)\right\rangle_{L^{2}}=i_{u} \partial_{y}\langle\mathcal{S}(y), \mathcal{S}(y)\rangle_{L^{2}}-\left\langle\nabla_{\bar{u}^{H}}^{E} \mathcal{S}(y), \mathcal{S}(y)\right\rangle_{L^{2}}
$$

where the last term vanishes because the section is holomorphic. Here we crucially used the fact that Lie derivative in direction $u^{H}$ of the volume form $L_{u^{H}} \omega_{\Sigma_{y}}=0$, as explained in Appendix A, Eq. (111). Then the statement of Eq. (79) follows. We can also write the connection Eq. (79) in the form

$$
\mathcal{A}_{y}=\frac{i}{2} \partial_{y} \log Z_{k}
$$

The proof is complete.

From the Eq. (84) above we arrive at the formula, which relates adiabatic curvature to the generating functional,

$$
\Omega=d_{Y} \mathcal{A}=-\partial_{Y} \bar{\partial}_{Y} \log Z_{k}
$$

The adiabatic curvature $\Omega$ is a closed $(1,1)$-form on the parameter space $Y$. The integrals of $\Omega$ over closed 2-cycles in $Y$ (5) are the adiabatic transport coefficients. The crucial physical observation, that goes back to [64, 54, 5], attributes the quantization of the Hall conductance to the fact that $\frac{i}{2 \pi} \Omega$ belongs to an integral cohomology class if $Y=\operatorname{Jac}(\Sigma)$. Another observation due to [6, 48, 49, 43] is that for $Y=\mathcal{M}_{\mathrm{g}}$ the curvature $\frac{i}{2 \pi} \Omega$ belongs to a rational cohomology class and defines geometric transport on the moduli space of complex structures.

Note that following formula holds

$$
\Omega=\Omega^{\mathcal{L}}-\partial_{Y} \bar{\partial}_{Y} \log \operatorname{det}^{\prime} \Delta_{\mathrm{L}}
$$

where $\Omega^{\mathcal{L}}=\partial_{Y} \bar{\partial}_{Y} \log \|\mathcal{S}\|^{2}$ is the curvature of Chern connection of the Quillen metric (43) on the determinant line bundle $\mathcal{L}$. The last term in (86), is in fact an exact form on $Y$, since $\operatorname{det}^{\prime} \Delta_{\mathrm{L}}$ is a function on $Y$. It vanishes under the integration over a closed 2-cycle, in which case the only contribution to the transport coefficients comes from $\Omega^{\mathcal{L}}$.

Now we recall the curvature formula, following [18], see also [16, 22]. We also note that this formula appeared in the QHE context in Ref. [63]. We denote the curvature of the metric $h^{E}$ on $E$ over $M$ by $\mathrm{F}^{E}$. In the same way we can extend the tangent bundle $T \Sigma$ to the union $T M \mid Y$ (note that this bundle is one-dimensional while $T M$ is not) over $M$, and let $g^{T M \mid Y}$ be any smooth Hermitian metric on $T M \mid Y$ (which is automatically Kähler along fibers since they are one-dimensional) with $R_{T M \mid Y}$ being its curvature 2-form. 
Then the following formula for the curvature of the determinant line bundle $\Omega^{\mathcal{L}}$ holds [18, Theorems 1.9, 1.27]

$$
\Omega^{\mathcal{L}}=-2 \pi i \int_{M \mid Y}[\operatorname{Ch}(E) \operatorname{Td}(T M \mid Y)]_{(4)} .
$$

Here the integrand is a form of mixed degree on $M$. The subscript 4 means that only 4 -form component of the full expression is retained, so that the result of the integration is a 2-from. The notation $M \mid Y$ means that the integration goes over the fibers in the fibration $\sigma: M \rightarrow Y$, i.e., over the spaces $\Sigma_{y}$ at $y$ fixed.

In order to apply [18, Theorem 1.27] we need to check that our fibration $\sigma: M \rightarrow Y$ with Riemann surfaces is locally Kähler. This can be seen as follows. Let $L$ be a holomorphic line bundle on $M$ which is positive along each fiber $\Sigma_{y}$ (for example, we can take $\left.L\right|_{\Sigma_{y}}=K_{\Sigma_{y}}$ if the genus of $\Sigma$ satisfies $g>1$ ). By taking a sufficiently large power of $L$, we can assume that $\operatorname{deg}\left(\left.L\right|_{\Sigma_{y}}\right)>2(1-\mathrm{g})+2$ for every $y \in Y$. Then by [38, p. 215], the Kodaira map $\Sigma_{y} \rightarrow \mathbb{P}\left(H^{0}\left(\Sigma_{y},\left.L\right|_{\Sigma_{y}}\right)^{*}\right)$ along the fiber $\Sigma_{y}$ is a holomorphic embedding for each $y \in Y$. These Kodaira maps vary holomorphically with $y \in Y$ and induce a holomorphic embedding of $M$ onto the total space of the holomorphic vector bundle $\mathbb{P}\left(H^{0}\left(\Sigma,\left.L\right|_{\Sigma}\right)^{*}\right)$ over $Y$, whose fibers are $\mathbb{P}\left(H^{0}\left(\Sigma_{y},\left.L\right|_{\Sigma_{y}}\right)^{*}\right)$. A smooth metric on the holomorphic vector bundle $H^{0}\left(\Sigma,\left.L\right|_{\Sigma}\right)$ on $Y$, it induces a smooth metric $h^{\mathcal{O}(1)}$ on the fiberwise hyperplane line bundle $\mathcal{O}(1)$ on $\mathbb{P}\left(H^{0}\left(\Sigma,\left.L\right|_{\Sigma}\right)^{*}\right)$. Now the restriction of the first Chern form of the Chern connection of $\left(\mathcal{O}(1), h^{\mathcal{O}(1)}\right)$ on $M$ gives a closed $(1,1)$-form which is Kähler along each fiber $\Sigma_{y}$. This shows that $\sigma: M \rightarrow Y$ is actually a Kähler fibration [17, Definition 1.4], cf. Appendix A.

5.2. Proof of Theorem 3. Let us write $\Omega^{\mathcal{L}}$ in Eq. (87) locally as an exterior derivative of a 1-form on $Y: \Omega^{\mathcal{L}}=d_{Y} \mathcal{A}^{\mathcal{L}}$. Then we choose an adiabatic process, i.e., a smooth closed contour $\mathcal{C}$ in $Y$. When the system is transported along the contour, the geometric part of the adiabatic phase (associated with Quillen metric) is given by the integral of the connection along $\mathcal{C}$

$$
\int_{\mathcal{C}} \mathcal{A}^{\mathcal{L}}
$$

Now we would like to obtain an explicit formula for this integral. The goal is to show that it is given by $(2+1)$ d integral of a specific Chern-Simons form over $\sigma^{-1}(\mathcal{C})$.

Let us focus on the structure of the integrand in (87). The Chern character form $\mathrm{Ch}(E)$ of a vector bundle $E \rightarrow M$ and the Todd form $\operatorname{Td}(T M)$ of the tangent bundle of a manifold $M$ are defined by the formal expansions in the powers of the corresponding curvature forms. Here we will need only a few first terms in these expansions

$$
\begin{aligned}
& \operatorname{Ch}(E)=1+c_{1}(E)+\frac{1}{2}\left(c_{1}^{2}(E)-2 c_{2}(E)\right)+\ldots \\
& \operatorname{Td}(T M)=1+\frac{1}{2} c_{1}(T M)+\frac{1}{12}\left(c_{1}^{2}(T M)+c_{2}(T M)\right)+\ldots
\end{aligned}
$$

\footnotetext{
${ }^{4}$ In the literature, there exist two different curvature formulas. In the smooth category, for a family of Dirac operators, Bismut-Freed defined in [16. Theorem 1.21] a unitary connection on the smooth determinant line bundle and computed its curvature. In the holomorphic category which we use here, Bismut-Gillet-Soulé [18. Theorem 1.27] computed the curvature of the Chern connection of a holomorphic determinant line bundle for a holomorphic locally Kähler fibration [18, Definition 1.25] with compact fiber. All curvatures in (87) are curvatures of associated Chern connections, and thus the integral of the right hand side of (87) is a (1,1)-form on the base manifold.
} 
where the Chern forms are given by

$$
\begin{aligned}
& c_{1}(E)=\frac{i}{2 \pi} \operatorname{Tr} \mathrm{F}^{E}, \quad c_{2}(E)=\frac{1}{8 \pi^{2}}\left(\operatorname{Tr} \mathrm{F}^{E} \wedge \mathrm{F}^{E}-\operatorname{Tr} \mathrm{F}^{E} \wedge \operatorname{Tr} \mathrm{F}^{E}\right), \\
& c_{1}(T M)=\frac{i}{2 \pi} \operatorname{Tr} \mathrm{R}^{T M}, \quad c_{2}(T M)=\frac{1}{8 \pi^{2}}\left(\operatorname{Tr} \mathrm{R}^{T M} \wedge \mathrm{R}^{T M}-\operatorname{Tr} \mathrm{R}^{T M} \wedge \operatorname{Tr} \mathrm{R}^{T M}\right) .
\end{aligned}
$$

In our case the 2 -forms $\mathrm{F}^{E}$ and $\mathrm{R}_{T M \mid Y}$ are scalar valued, so the traces shall be omitted, hence $c_{2}(E)=c_{2}(T M \mid Y)=0$. Also we split the curvature 2-form of the bundle $E$ as: $\mathrm{F}^{E}=$ $\mathrm{F}-s \mathrm{R}_{T M \mid Y}$ where $\mathrm{F}$ now refers to the part of the curvature 2-form corresponding to the line bundle $\tilde{L}^{k} \rightarrow M$, which is the union of all bundles $L_{y}^{k} \rightarrow \Sigma_{y}$. Using the composition property of the Chern character forms $\mathrm{Ch}\left(E \otimes E^{\prime}\right)=\mathrm{Ch}(E) \cdot \mathrm{Ch}\left(E^{\prime}\right)$ for any two bundles $E$, $E^{\prime}$, we get $\operatorname{Ch}\left(L^{k} \otimes K^{s}\right)=1+c_{1}\left(L^{k}\right)-s c_{1}(T M \mid Y)-s c_{1}\left(L^{k}\right) c_{1}(T M \mid Y)+\frac{1}{2}\left(c_{1}^{2}\left(L^{k}\right)+s^{2} c_{1}^{2}(T M \mid Y)\right)+\ldots$ Then the formula (87) specified to our case reads

$$
\Omega^{\mathcal{L}}=\frac{i}{4 \pi} \int_{M \mid Y}\left[\mathrm{~F} \wedge \mathrm{F}+(1-2 s) \mathrm{F} \wedge \mathrm{R}_{T M \mid Y}+\left(\frac{(1-2 s)^{2}}{4}-\frac{1}{12}\right) \mathrm{R}_{T M \mid Y} \wedge \mathrm{R}_{T M \mid Y}\right] .
$$

The holonomy $\exp \left(-\int_{\mathcal{C}} \mathcal{A}^{\mathcal{L}}\right)$ is the parallel transport operator from the point $\mathcal{C}_{0}$ into $\mathcal{C}_{1}\left(=\mathcal{C}_{0}\right)$ along the path $\mathcal{C}_{s}$ for the Chern connection on $(\mathcal{L},\|\cdot\|)$. It is a complex number of norm 1 and it does not depend on the origin $\mathcal{C}_{0}$. If $\mathcal{C}$ is the boundary of a surface $G$, then by (87),

$$
\exp \left(-\int_{\mathcal{C}} \mathcal{A}^{\mathcal{L}}\right)=\exp \left(2 i \pi \int_{\sigma^{-1}(G)}[\operatorname{Ch}(E) \operatorname{Td}(T M \mid Y)]_{(4)}\right) .
$$

By [18, Theorem 1.15], the unitary connection in [16] associated with the horizontal bundle (the orthogonal bundle to the vertical bundle) is exactly the Chern connection on $(\mathcal{L},\|\cdot\|)$, thus by [16, Theorem 3.16],

$$
\exp \left(-\int_{\mathcal{C}} \mathcal{A}^{\mathcal{L}}\right)=\exp (2 i \pi \bar{\eta})
$$

where $\bar{\eta}$ is the adiabatic limit of the reduced eta invariant for the fibration $\sigma: \sigma^{-1}(\mathcal{C}) \rightarrow \mathcal{C}$. Note that in [16, Theorem 3.16], the line bundle is the dual of our $\mathcal{L}$ and the spin structure of $\mathcal{C}$ (identified with $S^{1}$ ) is trivial. In our situation the spin structure of $\mathcal{C}$ is non-trivial, due to the choice of spinor $\Lambda\left(T^{*(0,1)} \sigma^{-1}(\mathcal{C})\right) \otimes E$, with $E=L^{k} \otimes K^{s}$, in Section 5.1. Thus the factor $(-1)^{\operatorname{Ind} D_{+}}$from [16, (3.164)] does not appear in (93). Moreover, to apply [18, Theorem 1.15], we assume the metric $g$ on $T \Sigma$ is the restriction of a Kähler metric on a neighborhood of $\sigma^{-1}(\mathcal{C})$. For a detailed discussion on how to combine Bismut-Gillet-Soulé's curvature formula and Bismut-Freed's holonomy theorem to study the holonomy of the determinant line bundle even for a singular fibration see [14, §6].

The eta invariant (form) is closely related to Chern-Simons theory. Let $E$ be a complex vector bundle on a manifold $X$. Let $\nabla^{E}$ be a connection on $E$, with curvature $\mathrm{F}^{E}=\left(\nabla^{E}\right)^{2} \in$ $\Omega^{2}(X, \operatorname{End}(E))$. For any real polynomial $q \in \mathbb{R}[z]$, we set

$$
Q\left(\mathrm{~F}^{E}\right)=\operatorname{Tr}\left[q\left(\frac{\sqrt{-1}}{2 \pi} \mathrm{F}^{E}\right)\right] \in \Omega^{\bullet}(X, \mathbb{C}) .
$$

By the Chern-Weil theory [51, Theorem B.5.1], $Q\left(\mathrm{~F}^{E}\right)$ is a closed differential form and its de Rham cohomology class $\left[Q\left(\mathrm{~F}^{E}\right)\right] \in H^{2 \bullet}(X, \mathbb{C})$ does not depend on the choice of $\nabla^{E}$. 
The Chern-Simons class $\widetilde{Q}\left(\nabla_{0}^{E}, \nabla_{1}^{E}\right) \in \Omega^{2 \bullet-1}(X, \mathbb{C}) / d \Omega^{\bullet}(X, \mathbb{C})$ associated to two connections $\nabla_{0}^{E}$ and $\nabla_{1}^{E}$ on $E$ is well defined (cf. [51, Theorem B.5.4]), and

$$
d \widetilde{Q}\left(\nabla_{0}^{E}, \nabla_{1}^{E}\right)=Q\left(\mathrm{~F}_{1}^{E}\right)-Q\left(\mathrm{~F}_{0}^{E}\right) .
$$

Let's go back to the context of (93). Let $g_{1}, h_{1}^{L}$ be another couple of metrics on $T \Sigma$, $L$, and $\bar{\eta}_{1}$ be the adiabatic limit of the associated reduced eta invariant. Then by [16, (3.196)], by applying [14, (6.33)-(6.36)] for the fibration $\sigma^{-1}(\mathcal{C}) \times[0,1] \rightarrow \mathcal{C} \times[0,1]$, we have modulo $\mathbb{Z}$

$$
\bar{\eta}-\bar{\eta}_{1}=\int_{\sigma^{-1}(\mathcal{C})} \text { Chern-Simons classes associated with two pairs } g, h \text { and } g_{1}, h_{1}^{L} \text {. }
$$

More precisely, let $g_{t}, h_{t}^{L}(t \in[0,1])$ be a path of metrics from $g, h$ to $g_{1}, h_{1}^{L}$. Let $A_{t}=$ $\partial \log \left(\left(h^{k}\right)^{-1} h_{t}^{L^{k}}\right)$, and $B_{t}=\partial \log \left((g)^{-1} g_{t}\right)$, and $F_{0}, R_{0}$ the curvatures of the Chern connections on $\left(L, h^{k}\right),(T \Sigma, g)$, then modulo $2 \pi i \mathbb{Z}$,

$$
\begin{aligned}
2 \pi i\left(\bar{\eta}-\bar{\eta}_{1}\right)=-\frac{i}{4 \pi} \int_{\sigma^{-1}(\mathcal{C})} \int_{0}^{1} d t & {\left[2\left(\frac{\partial}{\partial t} A_{t}\right)\left(\mathrm{F}_{0}+d A_{t}\right)\right.} \\
& +(1-2 s)\left(\left(\frac{\partial}{\partial t} A_{t}\right) \wedge\left(\mathrm{R}_{0}+d B_{t}\right)+\left(\mathrm{F}_{0}+d A_{t}\right) \wedge\left(\frac{\partial}{\partial t} B_{t}\right)\right) \\
& \left.+2\left(\frac{(1-2 s)^{2}}{4}-\frac{1}{12}\right)\left(\frac{\partial}{\partial t} B_{t}\right) \wedge\left(\mathrm{R}_{0}+d B_{t}\right)\right] \\
=\frac{i}{4 \pi} \int_{\sigma^{-1}(\mathcal{C})} A \wedge d A & +\frac{1-2 s}{2}(A \wedge d \omega+d A \wedge \omega)+\left(\frac{(1-2 s)^{2}}{4}-\frac{1}{12}\right) \omega \wedge d \omega \\
-\left(A \rightarrow A_{1}, \omega\right. & \left.\rightarrow \omega_{1}\right) .
\end{aligned}
$$

This ends the proof of Theorem 3 .

Remarks: Let us briefly recall the classical Chern-Simons functional. Let $Z$ be a manifold of dimension $\leq 3$. Then any principal $G$-bundle of a simply connected compact Lie group $G$ over $Z$ is trivializable. Assume also the structure group of $E$ is a simply connected compact Lie group, then $E$ is a trivial vector bundle on $Z$, and once we fix a trivialization of $E$, it induces a connection $\nabla_{0}^{E}$ which is the usual differential $d$. Then we denote $\widetilde{Q}\left(\nabla_{0}^{E}, \nabla_{1}^{E}\right)$ simply by $\widetilde{Q}\left(\nabla_{1}^{E}\right)$. In particular, if $Z$ is an oriented 3-dimensional manifold, this implies that the tangent bundle $T Z$ is trivial and we fix a trivialization of $T Z$. The classical Chern-Simons functional associated with the connection $\nabla^{T Z}=d+A$ is [51, Example B.5.7]

$$
C S\left(\nabla^{T Z}\right)=\int_{Z} \widetilde{p}_{1}\left(d, \nabla^{T Z}\right)=\frac{-1}{8 \pi^{2}} \int_{Z} \operatorname{Tr}\left[A \wedge d A+\frac{2}{3} A \wedge A \wedge A\right] \in \mathbb{R},
$$

where $p_{1}$ is the first Pontryagin form. Once we use another trivialization of $T Z, C S\left(\nabla^{T Z}\right)$ can change by an integer, thus $C S\left(\nabla^{T Z}\right) \in \mathbb{R} / \mathbb{Z}$ does not depend on the trivialization of $T Z$ and it is a well-defined functional on the space of connections on $T Z$.

However, in the context of our paper, for $Z=\sigma^{-1}(\mathcal{C})$, the associated Lie group is $S^{1}$ which is not simply connected. The line bundles $L$ and $T \Sigma$ are not trivial on $Z$, thus the last two 
terms in (96) are not well-defined Chern-Simons classes, but their difference is well defined on $\sigma^{-1}(\mathcal{C})$.

We show here how to define rigorously the last two terms in (96). By [41, §VII, Theorem 2], any connected orientable compact 3-dimensional manifold $Z$ bounds a simply connected orientable 4-dimensional manifold $W$. This implies that any smooth complex line bundle on $Z$ can be extended to a smooth complex line bundle on $W$.

Now let $W$ be such a 4-dimensional manifold with boundary the 3-dimensional manifold $Z=\sigma^{-1}(\mathcal{C})$. We extend the line bundles $L$ and $K_{\Sigma}^{1 / 2}$ from $\sigma^{-1}(\mathcal{C})$ to line bundles on $W$ denoted by $L$ and $K^{1 / 2}$, respectively. We extend also the connections of $L, K_{\Sigma}^{1 / 2} \rightarrow Z$ to connections of $L, K^{1 / 2} \rightarrow W$ such that they have a product structure near the boundary $Z$, that is, on a neighborhood $Z \times[0,1]$ of $Z$ in $W$, they are pull-back of the corresponding connections on $Z$. We denote the curvatures of $L$ and $K^{1 / 2}$ by $F$ and $-\frac{1}{2} R$, respectively. Inspired by [71, (2.3)], we can simply define the Chern-Simons functional by

$$
C S(A, \omega)=-\frac{1}{8 \pi^{2}} \int_{W}\left[\mathrm{~F} \wedge \mathrm{F}+(1-2 s) \mathrm{F} \wedge \mathrm{R}+\left(\frac{(1-2 s)^{2}}{4}-\frac{1}{12}\right) \mathrm{R} \wedge \mathrm{R}\right] .
$$

Then $C S(A, \omega)$ is well-defined modulo $\frac{1}{6} \mathbb{Z}$, i.e., it does not depend on the choice of $W$ and the extension of the line bundles and connections modulo $\frac{1}{6} \mathbb{Z}$. Let $\left(W^{\prime}, L, K^{1 / 2}\right)$ be another triple of extensions (with connections). Then $W \cup\left(-W^{\prime}\right)$ is a closed manifold (where $-W^{\prime}$ has the same underlying space as $W^{\prime}$ but the opposite orientation) and $L, K^{1 / 2}$ glue together to complex line bundles on $W \cup\left(-W^{\prime}\right)$ with induced smooth connections and curvatures $F$ and $-\frac{1}{2} R$, thus

$$
\begin{aligned}
& -\frac{1}{4 \pi^{2}} \int_{W \cup\left(-W^{\prime}\right)} \mathrm{F} \wedge \mathrm{F}=\int_{W \cup\left(-W^{\prime}\right)} c_{1}(L)^{2} \in \mathbb{Z}, \\
& -\frac{1}{8 \pi^{2}} \int_{W \cup\left(-W^{\prime}\right)} \mathrm{F} \wedge \mathrm{R}=\int_{W \cup\left(-W^{\prime}\right)} c_{1}(L) c_{1}\left(K^{1 / 2}\right) \in \mathbb{Z}, \\
& -\frac{1}{16 \pi^{2}} \int_{W \cup\left(-W^{\prime}\right)} \mathrm{R} \wedge \mathrm{R}=\int_{W \cup\left(-W^{\prime}\right)} c_{1}\left(K^{1 / 2}\right)^{2} \in \mathbb{Z} .
\end{aligned}
$$

Thus for $s \in \frac{1}{2} \mathbb{Z}, C S(A, \omega)$ is well-defined modulo $\frac{1}{6} \mathbb{Z}$.

Note that by removing the term $-\frac{1}{12} R \wedge R$ from the right-hand side of (98) the resulting expression is well defined modulo $\frac{1}{2} \mathbb{Z}$.

Note also that if $W$ and $W^{\prime}$ are fibrations of Riemann surfaces over surfaces $G, G^{\prime}$ with boundary $\mathcal{C}$, we can choose $\left(K^{1 / 2}\right)^{2}$ as the cotangent bundle of the Riemann surfaces. Then we can apply the Atiyah-Singer family index theorem for the fibration $\sigma: W \cup\left(-W^{\prime}\right) \rightarrow G \cup G^{\prime}$, and we have

$$
\frac{1}{8 \pi^{2}} \int_{W \cup\left(-W^{\prime}\right)}\left[\mathrm{F} \wedge \mathrm{F}+(1-2 s) \mathrm{F} \wedge \mathrm{R}+\left(\frac{(1-2 s)^{2}}{4}-\frac{1}{12}\right) \mathrm{R} \wedge \mathrm{R}\right] \in \mathbb{Z} .
$$

We can reformulate (96) rigorously as

$$
\bar{\eta}-\bar{\eta}_{1}=C S(A, \omega)-C S\left(A_{1}, \omega_{1}\right) \text { modulo } \frac{1}{6} \mathbb{Z} .
$$


Finally, we shall also add that it would be interesting to understand whether we have individually in (101),

$$
\bar{\eta}=C S(A, \omega) \text { modulo } \frac{1}{6} \mathbb{Z}
$$

In summary, we have derived the adiabatic phase starting from the $2 \mathrm{~d}$ QH-state and its generating functional, and using the Bismut-Gillet-Soulé curvature formula for Quillen metric. This observation establishes the link between $2 \mathrm{~d}$ [42, 25, 34, 26, 43] and $(2+1) \mathrm{d}$ [1, 39, 40, 68, 37] approaches to QHE.

\section{Appendix A: QUiLlen METRIC AND ANALYTIC TORSION}

Here we will review the basic facts on the Quillen metric cf. [51, §5.5]. Let $X$ be a compact complex manifold and let $E$ be a holomorphic vector bundle on $X$. Let $\Omega^{(0, \bullet)}(X, E)=$ $\bigoplus_{j} \Omega^{(0, j)}(X, E)$ be the space of smooth anti-holomorphic forms on $X$ with values in $E$. The holomorphic structure on $E$ allows us to define the Cauchy-Riemann operators $\bar{\partial}^{E}$ as follows. Any section $s \in \mathcal{C}^{\infty}(X, E)$ has the local form $s=\sum_{l} \varphi_{l} \xi_{l}$ where $\left\{\xi_{l}\right\}_{l=1}^{m}$ is a local holomorphic frame of $E$ and $\varphi_{l}$ are smooth functions. We set $\bar{\partial}^{E} s=\sum_{l}\left(\bar{\partial} \varphi_{l}\right) \xi_{l}$, where $\bar{\partial} \varphi_{l}=\sum_{j} d \bar{z}_{j} \frac{\partial}{\partial \bar{z}_{j}} \varphi_{l}$ in holomorphic coordinates $\left(z_{1}, \cdots, z_{n}\right)$. We define the $\bar{\partial}^{E}$-operator on $\Omega^{(0, \bullet)}(X, E)$ by setting $\bar{\partial}^{E}(\alpha \otimes s)=\bar{\partial} \alpha \otimes s+(-1)^{\operatorname{deg} \alpha} \alpha \wedge \bar{\partial}^{E} s$, where $\alpha \in \Omega^{(0, \bullet)}(U)$ and $s \in \mathcal{C}^{\infty}(U, E)$ for some open set $U \subset X$. We have then $\left(\bar{\partial}^{E}\right)^{2}=0$. Thus we get thus the Dolbeault complex $\left(\Omega^{(0, \bullet)}(X, E), \bar{\partial}^{E}\right)$ whose cohomology is called Dolbeault cohomology,

$$
H^{(0, \bullet)}(X, E)=\bigoplus_{j=0}^{n} H^{(0, j)}(X, E) \quad \text { with } H^{(0, j)}(X, E)=\left.\operatorname{ker} \bar{\partial}^{E}\right|_{\Omega^{(0, j)}} /\left.\operatorname{Im} \bar{\partial}^{E}\right|_{\Omega^{(0, j-1)}} .
$$

We denote it simply by $H^{\bullet}(X, E):=\bigoplus_{j} H^{j}(X, E):=\bigoplus_{j=0}^{n} H^{(0, j)}(X, E)$.

Let $h^{T X}$ be a Hermitian metric on the holomorphic tangent bundle $T X$ and $h^{E}$ a Hermitian metric on $E$. They induce an $L^{2}$-inner product $\langle\cdot, \cdot\rangle$ on $\Omega^{(0, \bullet)}(X, E)$ as in (30). Let $\bar{\partial}^{E *}$ be the adjoint of $\bar{\partial}^{E}$ with respect to this $L^{2}$-inner product. The Kodaira Laplacian $\square^{E}$ is defined by

$$
\square^{E}=\left(\bar{\partial}^{E}+\bar{\partial}^{E *}\right)^{2}=\bar{\partial}^{E *} \bar{\partial}^{E}+\bar{\partial}^{E} \bar{\partial}^{E *}: \Omega^{(0, j)}(X, E) \rightarrow \Omega^{(0, j)}(X, E) .
$$

By Hodge theory, the map ker $\left.\square^{E}\right|_{\Omega^{(0, j)}} \rightarrow H^{j}(X, E), \sigma \mapsto[\sigma]$, which sends a harmonic form to its Dolbeault cohomology class is an isomorphism ker $\left.\square^{E}\right|_{\Omega^{(0, j)}} \simeq H^{j}(X, E)$. We denote by $\square^{E,>0}$ the restriction of $\square^{E}$ to the orthogonal complement of ker $\square^{E}$. Then for $u \in \mathbb{C}$, $\operatorname{Re}(u)>n$, the operator $\left(\square^{E,>0}\right)^{-u}$ is a trace class operator and the theta function

$$
\theta(u)=-\left.\sum_{j=0}^{n}(-1)^{j} j \operatorname{Tr}\right|_{\Omega^{(0, j)}}\left[\left(\square^{E,>0}\right)^{-u}\right]
$$

extends as a meromorphic function on $\mathbb{C}$ which is holomorphic at 0 . This is a simple application of the small time asymptotic expansion of the heat kernel and the Mellin transformation. The Ray-Singer analytic torsion is defined as

$$
T=\exp \left(-\frac{1}{2} \frac{\partial \theta}{\partial u}(0)\right)
$$


The determinant line of the cohomology $H^{\bullet}(X, E)$ is the complex line given by

$$
\operatorname{det} H^{\bullet}(X, E)=\bigotimes_{j=0}^{n}\left(\operatorname{det} H^{j}(X, E)\right)^{(-1)^{j}},
$$

where for $F$ a complex vector space, $\operatorname{det} F=\Lambda^{\max } F$ is the complex line given by the exterior algebra of maximum degree of $F$ and $(\operatorname{det} F)^{-1}$ the dual line of $\operatorname{det} F$. We define

$$
\lambda(E)=\left(\operatorname{det} H^{\bullet}(X, E)\right)^{-1} .
$$

Let $h^{H(X, E)}$ be the $L^{2}$-metric on $H^{\bullet}(X, E)$ induced by the $L^{2}$-scalar product on $\Omega^{0, \bullet}(X, E)$. via the Hodge theory. Let $|\cdot|_{\lambda(E)}$ be the $L^{2}$-metric on $\lambda(E)$ induced by $h^{H(X, E)}$.

Definition 2. The Quillen metric $\|\cdot\|_{\lambda(E)}$ on the complex line $\lambda(E)$ is defined by

$$
\|\cdot\|_{\lambda(E)}=|\cdot|_{\lambda(E)} \exp \left(-\frac{1}{2} \theta^{\prime}(0)\right) .
$$

The Quillen metric $\|\cdot\|_{\lambda(E)}$ depends on the choice of the metrics $h^{T X}$ and $h^{E}$. If $h_{0}^{T X}, h_{1}^{T X}$ are two Kähler metrics on $T X$, and $h_{0}^{E}, h_{1}^{E}$ are two Hermitian metrics on $E$, let $\|\cdot\|_{\lambda(E), i}$ be the Quillen metric on $\lambda(E)$ associated with $\left(h_{i}^{T X}, h_{i}^{E}\right)$ for $i=0,1$. Bismut-Gillet-Soulé [18, Theorem 1.23] proved the anomaly formula, by expressing

$$
\log \frac{\|\cdot\|_{\lambda(E), 0}^{2}}{\|\cdot\|_{\lambda(E), 1}^{2}}
$$

as an integral of certain explicit computable local terms, the so-called Bott-Chern secondary classes. In particular, Theorem 2 in Section 4 is a special case of their result.

Let $\pi: M \rightarrow B$ be a holomorphic submersion of complex manifolds with compact fiber $X$, and let $E$ be a holomorphic vector bundle on $M$. Usually, the dimension of the fiberwise Dolbeault cohomology $H^{j}\left(X_{b},\left.E\right|_{X_{b}}\right)$ can jump dramatically for $b \in B$, thus it is not clear whether the family of complex lines $\lambda(E)_{b}$ parameterized by $b \in B$ can form a smooth line bundle on $B$.

Following a suggestion of Grothendieck, Knudsen-Mumford 44 solved the problem in 1976 for a projective map $\pi: M \rightarrow B$ (which means that there exists a holomorphic vector bundle $F$ over $B$ and a closed immersion $\imath: M \rightarrow \mathbb{P}(F)$ such that $\pi=p \circ \imath$, where $p: \mathbb{P}(F) \rightarrow B$ is the projectivisation of $F \rightarrow B$ ). They defined namely in this case a holomorphic line bundle $\lambda^{K M}$ over $B$ such that on $b \in B$, we have canonically $\lambda_{b}^{K M} \simeq \lambda(E)_{b}$.

By extending [56], Bismut-Freed [16] shown that we can define analytically a smooth structure of $\lambda(E)$ over $B$.

Assume now $\pi: M \rightarrow B$ is locally Kähler, i.e., if there exist an open covering $\mathcal{U}$ of $B$ such that for any $U \in \mathcal{U}$, there exists a Kähler metric on $\pi^{-1}(U)$. Bismut-Gillet-Soulé [18, $\S 1$ i)] defined a holomorphic structure on $\lambda(E)$ over $B$ such that $\lambda(E)$ is a holomorphic line bundle on $B$. Let $T X$ be the relative holomorphic tangent bundle of $\pi: M \rightarrow B$ and let $h^{T X}$ be a smooth Hermitian metric on $T X$ which induces a Kähler metric on each fiber $X$. Let $h^{E}$ be a Hermitian metric on $E$. Since the fiberwise cohomology group $H^{j}\left(X_{b},\left.E\right|_{X_{b}}\right)$ might jump, both the $L^{2}$-metric $|\cdot|_{\lambda(E)_{b}}$ on the line bundle $\lambda(E)$ and the analytic torsion $T_{b}$ need not be smooth over $B$. However, Bismut-Gillet-Soulé proved that the Quillen metric $\|\cdot\|_{\lambda(E)_{b}}$ forms a smooth metric on the line bundle $\lambda(E)$ over $B$, and in [18, Theorems 1.9, 1.27], they computed the curvature of the Chern connection of the holomorphic Hermitian line bundle $\left(\lambda(E),\|\cdot\|_{\lambda(E)}\right)$ 
over $B$ by using the local family Atiyah-Singer index theorem of Bismut [13]. If $\pi: M \rightarrow B$ is projective, they also proved that there is a canonical isomorphism $\lambda^{K M} \simeq \lambda(E)$ as holomorphic line bundles on $B$.

Let $\omega^{M}$ be a closed real $(1,1)$-form on $M$ such that its restriction along each fiber $X$ defines a hermitian metric $h^{T X}$ (In particular, $\pi: M \rightarrow B$ is locally Kähler). We assume that for each $j$, the dimension of $H^{j}\left(X_{b}, E_{X_{b}}\right)$ is constant on $b \in B$. Then $H^{j}\left(X_{b}, E_{X_{b}}\right)$ coincides with the fiber of the $j$-th direct image $R^{j} \pi_{*} E$ of the sheaf of holomorphic sections of $E$ via $\pi$, thus from the holomorphic structure on $R \pi_{*} E$, they form a holomorphic vector bundle $H^{j}(X, E)$ on $B$.

Let $\nabla^{H(X, E)}$ be the Chern connection on the holomorphic Hermitian vector bundle on $\left(H^{\bullet}(X, E)\right.$, $h^{H(X, E)}$ ) over $B$, cf. [51, Theorem 1.1.5]. A natural question is how to compute $\nabla^{H(X, E)}$ by using the Chern connection $\nabla^{E}$ on $\left(E, h^{E}\right)$. Observe that for any $s \in \mathcal{C}^{\infty}\left(B, H^{j}(X, E)\right)$, we can identify it as an element of $\mathcal{C}^{\infty}\left(M, \Lambda^{j}\left(T^{*(0,1)} X\right) \otimes E\right)$ via the Hodge theory.

Let $P_{b}: \Omega^{(0, \bullet)}\left(X_{b}, E\right) \rightarrow$ ker $\square^{E}$ be the fiberwise orthogonal projection for $b \in B$. Let $\nabla^{\Lambda\left(T^{*(0,1)} X\right) \otimes E}$ be the connection on $\Lambda\left(T^{*(0,1)} X\right) \otimes E$ induced by the Chern connections on $\left(T X, h^{T X}\right),\left(E, h^{E}\right)$. Let

$$
T_{\mathbb{R}}^{H} M:=\left\{V \in T_{\mathbb{R}} M: \omega^{M}(V, Y)=0 \text { for all } X \in T_{\mathbb{R}} X\right\} .
$$

Here we add a subscript $\mathbb{R}$ to indicate the underlying real vector bundles. Then $T_{\mathbb{R}} M=$ $T_{\mathbb{R}}^{H} M \oplus T_{\mathbb{R}} X$ and for $U \in T_{\mathbb{R}, b} B$, we denote by $U^{H} \in T_{\mathbb{R}}^{H} M$ the unique lift such that $d \pi\left(U^{H}\right)=U$. If $u \in T^{(1,0)} B$ then $u^{H} \in T^{(1,0)} M$. Then Bismut-Köhler [19, Theorem 3.5] proved that for any $s \in \mathcal{C}^{\infty}\left(B, H^{\bullet}(X, E)\right)$,

$$
\nabla_{U}^{H(X, E)} s=P \nabla_{U^{H}}^{\Lambda\left(T^{*(0,1)} X\right) \otimes E} s .
$$

In particular for $s \in \mathcal{C}^{\infty}\left(B, H^{0}(X, E)\right)$, we have

$$
\nabla_{U}^{H^{0}(X, E)} s=P \nabla_{U^{H}}^{E} s .
$$

To verify directly that the right hand side of (108) is a Hermitian connection, we need to verify that for $s_{1}, s_{2} \in \mathcal{C}^{\infty}\left(B, H^{\bullet}(X, E)\right)$

$$
U\left\langle s_{1}, s_{2}\right\rangle=\left\langle P \nabla_{U^{H}}^{\Lambda\left(T^{*(0,1)} X\right) \otimes E} s_{1}, s_{2}\right\rangle+\left\langle s_{1}, P \nabla_{U^{H}}^{\Lambda\left(T^{*(0,1)} X\right) \otimes E} s_{2}\right\rangle,
$$

but this is a consequence of the horizontal Lie derivative of the fiberwise volume form $\left(\left.\omega^{M}\right|_{X}\right)^{n} / n$ ! is zero, i.e.,

$$
L_{U^{H}}\left(\left.\omega^{M}\right|_{X}\right)^{n}=0
$$

which is implied by the closeness of $\omega^{M}$ cf. [17, Theorem 1.7, part d].

\section{Appendix B: Expansion of the Bergman keRnel on Riemann surfaces}

Here we review the asymptotic expansion of the Bergman kernel, following Ref. [51.

Let $(X, J)$ be a compact complex manifold of $\operatorname{dim}_{\mathbb{C}} X=n$. Let $\omega$ be a positive $(1,1)$-form on $X$, and we denote by $g^{T X}$ the Riemannian metric associated to $\omega$. Then $d v_{X}=\omega^{n} / n$ ! is the Riemannian volume form of $(X, \omega)$. Let $\nabla^{T X}$ be the Levi-Civita connection on $(X, \omega)$. We denote by $R^{T X}=\left(\nabla^{T X}\right)^{2}$ the curvature, by Ric the Ricci curvature and by $R$ the scalar curvature of $\nabla^{T X}$. Let $\operatorname{Ric}_{\omega}=\operatorname{Ric}(J \cdot, \cdot)$ be the $(1,1)$-form associated to Ric. Let $\Delta$ be the (negative) Laplace operator on $\left(X, g^{T X}\right)$ acting on the functions on $X$. 
Let $\left(L, h^{L}\right)$ and $\left(E, h^{E}\right)$ be holomorphic Hermitian vector bundles on $X$, with $\operatorname{rk} L=1$. On the space $\mathcal{C}^{\infty}\left(X, L^{k} \otimes E\right)$ of smooth sections of $L^{k} \otimes E$ we introduce an $L^{2}$-scalar product $\langle\cdot, \cdot\rangle$ given by

$$
\left\langle\sigma_{1}, \sigma_{2}\right\rangle:=\int_{X}\left\langle\sigma_{1}(x), \sigma_{2}(x)\right\rangle_{h^{L^{k}} \otimes h^{E}} d v_{X}(x) .
$$

We denote by $L^{2}\left(X, L^{k} \otimes E\right)$ the completion of $\mathcal{C}^{\infty}\left(X, L^{k} \otimes E\right)$ with respect to (112). We also introduce the space $H^{0}\left(X, L^{k} \otimes E\right)$ of holomorphic sections of $L^{k} \otimes E$ on $X$. Let

$$
P_{k}: L^{2}\left(X, L^{k} \otimes E\right) \longrightarrow H^{0}\left(X, L^{k} \otimes E\right)
$$

be the orthogonal projection, called Bergman projection. For $z, z^{\prime} \in X$ let $P_{k}\left(z, z^{\prime}\right)$ be the smooth kernel of $P_{k}$ with respect to $d v_{X}\left(x^{\prime}\right)$, that is,

$$
\left(P_{k} s\right)(z)=\int_{X} P_{k}\left(z, z^{\prime}\right) s\left(z^{\prime}\right) d v_{X}\left(z^{\prime}\right), \quad s \in L^{2}\left(X, L^{k} \otimes E\right) .
$$

Note that the $L^{2}$-scalar product $\langle\cdot, \cdot\rangle$ and hence the kernel $P_{k}(\cdot, \cdot)$ depend on the Hermitian metrics $h^{L}, h^{E}$ and $\omega$. Let $\left\{s_{j}\right\}_{j=1}^{d_{p}}$ be an orthonormal basis of $\left(H^{0}\left(X, L^{k} \otimes E\right),\langle\cdot, \cdot\rangle\right)$. If $E$ is a line bundle,

$$
P_{k}(x, x)=\sum_{k=1}^{d_{p}}\left|s_{k}(x)\right|_{h^{L^{k} \otimes h^{E}}}^{2} .
$$

For $\psi \in \mathcal{C}^{\infty}(X)$ and denote

$$
h_{\psi}^{L}:=h^{L} e^{-\psi} .
$$

We assume that the curvature form of $\left(L, h_{\psi}^{L}\right)$, cf. [51, Theorem 1.1.5]

$$
\omega_{\psi}:=\frac{i}{2 \pi} R_{\psi}^{L}:=\frac{i}{2 \pi} R^{L}+\frac{i}{2 \pi} \partial \bar{\partial} \psi
$$

is a Kähler metric on $X$.

We will associate to a $(1,1)$-form $\eta$ an endomorphism

$$
\dot{\eta} \in \operatorname{End}\left(T^{(1,0)} X\right), \eta(u, \bar{v})=\langle\dot{\eta}(u), v\rangle_{\omega} .
$$

Note that the volume forms corresponding to $\omega$ and $\omega_{\psi}$ are linked by

$$
\omega_{\psi}^{n} / n !=\left(\operatorname{det} \dot{\omega}_{\psi}\right) \omega^{n} / n !
$$

We consider the $L^{2}$-scalar product $\langle\cdot, \cdot\rangle$ on $\mathcal{C}^{\infty}\left(X, L^{k} \otimes E\right)$ constructed as in (112) but with the metrics $h_{\psi}^{L}$ on $L, h^{E}$ on $E$ and with respect to the volume form $\omega^{n} / n$ ! on $X$. We have then a corresponding Bergman projection $P_{k}$ as in (113). We denote by $P_{k}\left(x, x^{\prime}\right)$ the Schwartz kernel of $P_{k}$ with respect to the volume form $\omega^{n} / n$ ! (see (114)).

We consider moreover the $L^{2}$-scalar product $\langle\cdot, \cdot\rangle_{\psi}$ constructed as in (112) with the metrics $h_{\psi}^{L}$ on $L, h_{\psi}^{E}=\left(\operatorname{det} \dot{\omega}_{\psi}\right)^{-1} h^{E}$ on $E$ and with respect to the volume form $\omega_{\psi}^{n} / n$ ! on $X$. Note that $\langle\cdot, \cdot\rangle_{\psi}=\langle\cdot, \cdot\rangle$ so that the Bergman projection $P_{k}$ (cf. (113)) is the same for these scalar products. We denote $P_{k, \psi}\left(x, x^{\prime}\right)$ the Schwartz kernel by of $P_{k}$ with respect to $\omega_{\psi}^{n} / n$ !. By (119), we have (cf. [51, (4.1.114)] or [52, Remark 0.5])

$$
P_{k}\left(x, x^{\prime}\right)=\left(\operatorname{det} \dot{\omega}_{\psi}\right)\left(x^{\prime}\right) P_{k, \psi}\left(x, x^{\prime}\right) .
$$


The advantage of passing from $P_{k}$ to $P_{k, \psi}$ is that $\left(L, h_{\psi}^{L}\right)$ polarizes $\left(X, \omega_{\psi}\right)$, that is, $\frac{i}{2 \pi} R^{\left(L, h_{\psi}^{L}\right)}=$ $\omega_{\psi}$, cf. (117). We will use now the asymptotic expansion of the Bergman kernel function $P_{k, \psi}(x, x)$. For this purpose we introduce more notations.

In the sequel we will mainly work with the Kähler form $\omega_{\psi}$, in which case we will use a subscript $\psi$, e.g., $\operatorname{Ric}_{\psi}, \Delta_{\psi}$ etc. Then for any $f \in \mathcal{C}^{\infty}(X)$ we have $\left\langle\bar{\partial} \partial f, \omega_{\psi}\right\rangle_{\psi}=\frac{i}{2} \Delta_{\psi} f$. Set

$$
R_{\psi}^{E}=R^{\left(E, h_{\psi}^{E}\right)}, \quad R_{\psi, \Lambda}^{E}=\left\langle R_{\psi}^{E}, \omega_{\psi}\right\rangle_{\psi} .
$$

By [52, Theorem 0.1] (cf. [27], [73], [50] without the twist bundle E), we have the following asymptotics as $k \rightarrow \infty$,

$$
P_{k, \psi}(x, x)=k^{n}+\left(\frac{R_{\psi}}{8 \pi}+\frac{i}{2 \pi} R_{\psi, \Lambda}^{E}\right) k^{n-1}+\frac{1}{\pi^{2}}\left(\boldsymbol{b}_{2 \mathbb{C} \psi}+\boldsymbol{b}_{2 E \psi}\right) k^{n-2}+\mathcal{O}\left(k^{n-3}\right),
$$

where

$$
\begin{aligned}
\boldsymbol{b}_{2 \mathbb{C} \psi} & =\frac{\Delta_{\psi} R_{\psi}}{48}+\frac{1}{96}\left|R_{\psi}^{T X}\right|_{\psi}^{2}-\frac{1}{24}\left|\operatorname{Ric}_{\psi}\right|_{\psi}^{2}+\frac{1}{128} R_{\psi}^{2} \\
& =\frac{\Delta_{\psi} R_{\psi}}{48}+\frac{1}{6} R_{\psi, \bar{\jmath} m \bar{q}} R_{\psi, \bar{\ell} q \bar{m}}-\frac{2}{3} R_{\psi, \ell \bar{\ell} m \bar{q}} R_{\psi, \bar{j} \bar{j} \bar{m}}+\frac{1}{2} R_{\psi, \bar{\ell} q \bar{q}} R_{\psi, \bar{j} \bar{j} m \bar{m}}
\end{aligned}
$$

and

$$
\begin{aligned}
\boldsymbol{b}_{2 E \psi}= & \frac{i}{16}\left(R_{\psi} R_{\psi, \Lambda}^{E}-2\left\langle\mathrm{Ric}_{\psi}, R_{\psi}^{E}\right\rangle_{\psi}+\Delta_{\psi}^{E} R_{\psi, \Lambda}^{E}\right)-\frac{1}{8}\left(R_{\psi, \Lambda}^{E}\right)^{2}+\frac{1}{8}\left\langle R_{\psi}^{E}, R_{\psi}^{E}\right\rangle_{\psi} \\
& =R_{\psi, q \bar{q}}^{E} R_{\psi, j \bar{j} m \bar{m}}-R_{\psi, m \bar{q}}^{E} R_{\psi, j \bar{j} q \bar{m}}+\frac{1}{2}\left(R_{\psi, q \bar{q}}^{E} R_{\psi, m \bar{m}}^{E}-R_{\psi, m \bar{q}}^{E} R_{\psi, q \bar{m}}^{E}\right)+\frac{1}{2} R_{\psi, j \bar{j} ; m \bar{m}}^{E} .
\end{aligned}
$$

Here in normal coordinate associated with $\omega_{\psi}$ at $x_{0}$,

$$
\begin{aligned}
& \omega_{\psi}=\frac{i}{2} \sum_{j} d z_{j} \wedge d \bar{z}_{j}, \\
& R_{\psi, j \bar{m} \ell \bar{q}}=\left\langle R_{\psi}^{T X}\left(\frac{\partial}{\partial z_{j}}, \frac{\partial}{\partial \bar{z}_{m}}\right) \frac{\partial}{\partial z_{\ell}}, \frac{\partial}{\partial \bar{z}_{q}}\right\rangle_{\psi, x_{0}}, \quad R_{\psi, j \bar{\ell}}^{E}=R_{\psi, x_{0}}^{E}\left(\frac{\partial}{\partial z_{j}}, \frac{\partial}{\partial \bar{z}_{\ell}}\right), \\
& R_{\psi, j \bar{q} ; t \bar{s}}^{E}=\frac{\partial^{2}}{\partial z_{t} \partial \bar{z}_{s}} R_{\psi}^{E}\left(\frac{\partial}{\partial z_{j}}, \frac{\partial}{\partial \bar{z}_{q}}\right), \quad i R_{\psi, \Lambda}^{E}=2 R_{\psi, j \bar{j}}^{E} .
\end{aligned}
$$

From now on, we assume that $X=\Sigma$ is a (connected) Riemann surface with the Kähler form $\omega$. We use the local normal coordinate associated with $\omega$ near $x_{0}$, then at $x_{0}$,

$$
\omega=\frac{i}{2} d z \wedge d \bar{z}, \quad \Delta=4 \frac{\partial^{2}}{\partial z \partial \bar{z}}, \quad(\Delta \varphi) \omega=-2 i \bar{\partial} \partial \varphi .
$$

At $x_{0}$, the scalar curvature $R$ of $(X, \omega)$ is given by

Thus

$$
R=4 R^{T^{(1,0)} X}\left(\frac{\partial}{\partial z}, \frac{\partial}{\partial \bar{z}}\right) .
$$

$$
-\frac{i}{2} R \omega=R^{T^{(1,0)} X}=-R^{K_{X}}=\bar{\partial} \partial \log |\sigma|^{2},
$$

where $\sigma$ is a local holomorphic frame of $T^{(1,0)} X$. 
For the Kähler form $\omega_{\psi}$ in (117), by (126), we have

$$
\Delta_{\psi}=a^{-1} \Delta, \quad \text { with } a=\frac{\omega_{\psi}}{\omega} .
$$

Thus by (126), (128) and (129) we get $-\frac{i}{2} R_{\psi} \omega_{\psi}=-\frac{i}{2} R \omega+\bar{\partial} \partial \log a$, and

$$
R_{\psi}=a^{-1} R-\frac{1}{a} \Delta \log a .
$$

Observe that $\operatorname{deg} K_{X}$ is even, thus $K_{X}^{1 / 2}$ is well-defined. Now, for $s \in \frac{1}{2} \mathbb{Z}$, we take $E=K_{X}^{s}$ with metric $g^{\otimes s}$ induced by $\omega$, then $h_{\psi}^{E}=a^{-1} g^{\otimes s}$ and

$$
R_{\psi, \Lambda}^{E} \omega_{\psi}=R_{\psi}^{E}=R_{\psi}^{K_{X}^{s}}=s R^{K_{X}}-\bar{\partial} \partial \log a .
$$

In particular, by (126), (128) and (131), we get

$$
i a R_{\psi, \Lambda}^{E}=-\frac{s}{2} R+\frac{1}{2} \Delta \log a
$$

By (122), $P_{k, \psi}\left(x, x^{\prime}\right)$ has an asymptotic expansion with coefficients

$$
\frac{R_{\psi}}{8 \pi}+\frac{i}{2 \pi} R_{\psi, \Lambda}^{E}=\frac{1}{2 \pi}\left(\frac{1}{4} R_{\psi}-\frac{s}{2 a} R+\frac{1}{2 a} \Delta \log a\right)=\frac{1}{2 \pi}\left(-\frac{1}{4} R_{\psi}+\frac{-s+1}{2 a} R\right),
$$

and

$$
\begin{aligned}
\boldsymbol{b}_{2 \mathbb{C} \psi} & +\boldsymbol{b}_{2 E \psi}=\frac{\Delta_{\psi} R_{\psi}}{48}+\frac{i}{16} \Delta_{\psi} R_{\psi, \Lambda}^{E} \\
& =\frac{1}{24 a} \Delta\left(\frac{1}{2 a} R-\frac{1}{2 a} \Delta \log a\right)+\frac{1}{16 a} \Delta\left(-\frac{s}{2 a} R+\frac{1}{2 a} \Delta \log a\right) \\
& =\frac{1}{48 a} \Delta\left(\frac{-3 s+2}{2 a} R+\frac{1}{2 a} \Delta \log a\right) .
\end{aligned}
$$

Combining (120), (122), (129), (133) and (134) we obtain as $k \rightarrow \infty$,

$$
\begin{aligned}
P_{k}(x, x)= & a k+\frac{a}{2 \pi}\left(-\frac{1}{4} R_{\psi}+\frac{-s+1}{2 a} R\right) \\
& +\frac{1}{48 \pi^{2}} \Delta\left(\frac{-3 s+2}{2 a} R+\frac{1}{2 a} \Delta \log a\right) k^{-1}+\mathcal{O}\left(k^{-2}\right) .
\end{aligned}
$$

Setting now $\omega_{\psi}=a \omega, B=2 \pi k a$ and $m=s$, we arrive at the expression in Eq. (57).

\section{REFERENCES}

[1] A. G. Abanov and A. Gromov, Electromagnetic and gravitational responses of two-dimensional non-interacting electrons in background magnetic field, Phys. Rev. B 90 (2014) 014435, arXiv:1401.3703 [cond-mat.str-el].

[2] L. Alvarez-Gaume, G. Moore and C. Vafa, Theta functions, modular invariance, and strings, Comm. Math. Phys. 106 (1986) 1-40.

[3] M. F. Atiyah, V. K. Patodi and I. M. Singer, Spectral asymmetry and Riemannian geometry. II, Bull. London Math. Soc. 5 (1973) 229-234.

[4] M. F. Atiyah and I. M. Singer, The index of elliptic operators. IV, Ann. of Math. (2) 93 119-138 (1971).

[5] J. E. Avron and R. Seiler, Quantization of the Hall conductance for general, multiparticle Schrödinger hamiltonians, Phys. Rev. Lett. 54 (1985) 259. 
[6] J. E. Avron, R. Seiler and P. G. Zograf, Adiabatic quantum transport: quantization and fluctuations, Phys. Rev. Lett. 73 no. 24 (1994) 3255-3257.

[7] J. E. Avron, R. Seiler and P. G. Zograf, Viscosity of quantum Hall fluids, Phys. Rev. Lett. 75 no. 4 (1995) 697-700, arXiv: cond-mat/9502011.

[8] A. Belavin and V. Knizhnik, Algebraic geometry and the geometry of quantum strings, Phys. Lett. B 168 no. 3 (1986) 201-206; Complex geometry and the theory of quantum strings, Sov. Phys. JETP 64 no. 2 (1986) 215-228.

[9] N. Berline and E. Getzler and M. Vergne, Heat kernels and Dirac operators, Grundlehren der Mathematischen Wissenschaften 298. Springer-Verlag, Berlin, 1992. viii+369 pp.

[10] R. Berman, Kähler-Einstein metrics emerging from free fermions and statistical mechanics, JHEP 10 (2011) 106, arXiv:1009.2942 [hep-th].

[11] R. Berman, Determinantal point processes and fermions on complex manifolds: large deviations and bosonization, Comm. Math. Phys. 327 (2014) 1-47, arXiv:0812.4224 [math.CV].

[12] A. Berthomieu, Analytic torsion of all vector bundles over an elliptic curve. J. Math. Phys. 42 (2001), no. $9,4466-4487$.

[13] J.-M. Bismut, The Atiyah-Singer Index Theorem for families of Dirac operators: two heat equation proofs, Invent. Math. 83 (1986) 91-151.

[14] J.-M. Bismut and J.-B. Bost, Fibrés déterminants, métriques de Quillen et dégénérescence des courbes, Acta Math. 165 (1990), no. 1-2, 1-103.

[15] J.-M. Bismut and J. Cheeger, $\eta$-invariants and their adiabatic limits, J. Amer. Math. Soc. 2 (1989), no. $1,33-70$.

[16] J.-M. Bismut and D. Freed, The analysis of elliptic families. I-II, Comm. Math. Phys. 106 no. 1 (1986) 159-176; 107 no. 1 (1987) 103-163.

[17] J.-M. Bismut, H. Gillet and C. Soulé, Analytic torsion and holomorphic determinant bundles. II. Direct images and Bott-Chern forms, Comm. Math. Phys. 115 no. 1 (1988) 79-126.

[18] J.-M. Bismut, H. Gillet and C. Soulé, Analytic torsion and holomorphic determinant bundles. III. Quillen metrics on holomorphic determinants, Comm. Math. Phys. 115 no. 2 (1988) 301-351.

[19] J.-M. Bismut and K. Köhler, Higher analytic torsion forms for direct images and anomaly formulas, J. Algebraic Geom. 1 (1992), no. 4, 647-684.

[20] J.-M. Bismut and E. Vasserot, The asymptotics of the Ray-Singer analytic torsion associated with high powers of a positive line bundle, Comm. Math. Phys. 125 (1989) 355-367.

[21] J.-B. Bost, Intrinsic heights of stable varieties and abelian varieties. Duke Math. J. 82 (1996), no. 1, 21-70.

[22] J.-B. Bost and T. Jolicœur, A holomorphy property and the critical dimension in string theory from an index theorem, Nucl. Phys. B 286 (1987) 175-188.

[23] B. Bradlyn and N. Read, Low-energy effective theory in the bulk for transport in a topological phase, Phys. Rev. B 91 (2015) 125303, arXiv:1407.2911 [cond-mat.mes-hall].

[24] B. Bradlyn and N. Read, Topological central charge from Berry curvature: Gravitational anomalies in trial wave functions for topological phases, Phys. Rev. B 91 (2015) 165306, arXiv:1502.04126 [cond-mat.mes-hall].

[25] T. Can, M. Laskin and P. Wiegmann, Fractional quantum Hall effect in a curved space: gravitational anomaly and electromagnetic response, Phys. Rev. Lett. 113 (2014) 046803, arXiv:1402.1531 [cond-mat.str-el]

[26] T. Can, M. Laskin and P. Wiegmann, Geometry of quantum Hall states: Gravitational anomaly and transport coefficients, Ann. Phys. 362, 752-794 (2015) arXiv:1411.3105 [cond-mat.str-el].

[27] D. Catlin, The Bergman kernel and a theorem of Tian, Analysis and geometry in several complex variables (Katata, 1997), Trends Math., Birkhäuser Boston, Boston, MA, 1999, pp. 1-23.

[28] X. Dai, Adiabatic limits, nonmultiplicativity of signature, and Leray spectral sequence. J. Amer. Math. Soc., 4, 265-321, (1991).

[29] E. D'Hoker and D. H. Phong, On determinants of laplacians on riemann surfaces, Commun. Math. Phys. 104 (1986) 537-545.

[30] E. D'Hoker and D. H. Phong, The geometry of string perturbation theory, Rev. Mod. Phys. 60 (1988) 917. 
[31] S. K. Donaldson, Scalar curvature and projective embeddings. II, Q. J. Math. 56 no. 3 (2005) 345-356, arXiv:math/0407534 [math.DG].

[32] M. R. Douglas and S. Klevtsov, Bergman kernel from path integral, Comm. Math. Phys. 293 no. 1 (2010) 205-230, arXiv:0808.2451 [hep-th].

[33] J. Fay, Kernel functions, analytic torsion and moduli spaces, Memoirs of AMS, 96 no. 464, Providence RI (1992).

[34] F. Ferrari and S. Klevtsov, FQHE on curved backgrounds, free fields and large N, JHEP 12 (2014) 086 , arXiv:1410.6802 [hep-th].

[35] F. Ferrari, S. Klevtsov and S. Zelditch, Gravitational actions in two dimensions and the Mabuchi functional, Nucl. Phys. B 859 no. 3 (2012) 341-369, arXiv:1112.1352 [hep-th].

[36] P. J. Forrester, Log-gases and random matrices, Princeton University Press (2010).

[37] J. Fröhlich and U. M. Studer, $U(1) \times S U(2)$-gauge invariance of non-relativistic quantum mechanics, and generalized Hall effects, Comm. Math. Phys. 148 (1992) 553-600.

[38] P. Griffiths and J. Harris, Principles of Algebraic Geometry, John Wiley and Sons, New York, 1978.

[39] A. Gromov and A. G. Abanov, Density-curvature response and gravitational anomaly, Phys. Rev. Lett. 113 (2014) 266802, arXiv:1403.5809 [cond-mat.str-el].

[40] A. Gromov, G. Y. Cho, Y. You, A. G. Abanov and E. Fradkin, Framing anomaly in the effective theory of fractional quantum Hall effect, Phys. Rev. Lett. 114 (2015) 016805, arXiv:1410.6812 [cond-mat.str-el].

[41] R. Kirby, The topology of 4-manifolds. Lecture Notes in Mathematics, 1374. Springer-Verlag, Berlin, 1989, 108 pp.

[42] S. Klevtsov, Random normal matrices, Bergman kernel and projective embeddings, JHEP 1401 (2014) 133, arXiv:1309.7333 [hep-th].

[43] S. Klevtsov and P. Wiegmann, Geometric adiabatic transport in Quantum Hall states, Phys. Rev. Lett. 115 (2015) 086801, arXiv:1504.07198 [cond-mat.str-el].

[44] F. Knudsen and D. Mumford. The projectivity of the moduli space of stable curves. I. Preliminaries on 'det' and 'Div'. Math. Scand. 39 (1976) 19-55.

[45] K. Köhler, Holomorphic torsion on Hermitian symmetric spaces. J. Reine Angew. Math. 460 (1995), 93-116.

[46] M. Laskin, T. Can and P. Wiegmann, Collective field theory for quantum Hall states, arXiv:1412.8716 [cond-mat.str-el].

[47] R. B. Laughlin, Anomalous quantum Hall effect: an incompressible quantum fluid with fractionally charged excitations, Phys. Rev. Lett. 50 no. 18 (1983) 1395.

[48] P. Lévay, Berry phases for Landau Hamiltonians on deformed tori, J. Math. Phys. 36 (1995) 2792-2802.

[49] P. Lévay, Berry's phase, chaos, and the deformations of Riemann surfaces, Phys. Rev. E 56 no. 5 (1997) $6173-6176$.

[50] Z. Lu, On the lower order terms of the asymptotic expansion of Tian-Yau-Zelditch, Amer. J. Math. 122 (2000), no. 2, 235-273.

[51] X. Ma and G. Marinescu, Holomorphic Morse inequalities and Bergman kernels, Progress in Mathematics, 254. Birkhäuser Verlag, Basel, 2007. xiv+422 pp.

[52] X. Ma and G. Marinescu, Berezin-Toeplitz quantization on Kähler manifolds, J. Reine Angew. Math. 662 (2012) 1-56, arXiv:1009.4405 [math.DG].

[53] D. Mumford, Tata lectures on theta I, Birkhäuser, Boston (1983).

[54] Q. Niu, D. J. Thouless and Y.-S. Wu, Quantized Hall conductance as a topological invariant, Phys. Rev. B31 (1985) 3372.

[55] A. M. Polyakov, Quantum gravity in two dimensions, Mod. Phys. Lett. A 2 no. 11 (1987) 893-898.

[56] D. Quillen, Determinants of Cauchy-Riemann operators over a Riemann surface, Funct. Anal. Appl. 19 no. 1 (1985) 37-41.

[57] D. B. Ray and I. M. Singer, Analytic torsion for complex manifolds, Ann. of Math. (2) 98 154-177 (1973).

[58] N. Read, Non-Abelian adiabatic statistics and Hall viscosity in quantum Hall states and $p_{x}+i p_{y}$ paired superfluids, Phys. Rev. B 79 no. 4 (2009) 045308, arXiv:0805.2507 [cond-mat.mes-hall]. 
[59] N. Read and E. H. Rezayi, Hall viscosity, orbital spin, and geometry: Paired superfluids and quantum Hall systems, Phys. Rev. B 84 no. 4 (2009) 085316, arXiv:1008.0210 [cond-mat.mes-hall].

[60] B. Simon, Holonomy, the quantum adiabatic theorem, and Berry's phase, Phys. Rev. Lett. 51 (1983) 2167.

[61] D. T. Son, Newton-Cartan Geometry and the Quantum Hall Effect, arXiv:1306.0638 [cond-mat.mes-hall].

[62] R. Tao and Y.-S. Wu, Gauge invariance and fractional quantum Hall effect, Phys. Rev. B 30 (1984) 1097.

[63] C. Tejero Prieto, Fourier-Mukai transform and adiabatic curvature of spectral bundles for Landau Hamiltonians on Riemann surfaces, Comm. Math. Phys. 265 (2006), no. 2, 373-396.

[64] D. J. Thouless, M. Kohmoto, M. P. Nightingale and M. den Nijs, Quantized Hall conductance in a twodimensional periodic potential, Phys. Rev. Lett. 49 (1982) 405.

[65] I. V. Tokatly and G. Vignale, Lorentz shear modulus of a two-dimensional electron gas at high magnetic field, Phys. Rev. B76 (2007) 161305, arXiv:0706.2454 [cond-mat.mes-hall].

[66] I. Tokatly and G. Vignale, Lorentz shear modulus of fractional quantum Hall states, J. Phys. C 21 (2009) 275603, arXiv:0812.4331 [cond-mat.mes-hall],

[67] E. P. Verlinde and H. L. Verlinde, Chiral bosonization, determinants and the string partition function, Nucl. Phys. B288 (1987) 357-396.

[68] X. G. Wen and A. Zee, Shift and spin vector: New topological quantum numbers for the Hall fluids, Phys. Rev. Lett. 69 (1992) 953.

[69] L. Weng, Regularized determinants of Laplacians for Hermitian line bundles over projective spaces. J. Math. Kyoto Univ. 35 (1995), no. 3, 341-355.

[70] E. Witten, Global gravitational anomalies, Comm. Math. Phys. 100 no. 2 (1985) 197-229.

[71] E. Witten, $S L(2, \mathbb{Z})$ action on 3-dimensional conformal field theories with abelian symmetry. From fields to strings: circumnavigating theoretical physics. Vol. 2, 1173-1200, World Sci. Publ., Singapore, 2005.

[72] A. Zabrodin and P. Wiegmann, Large $N$ expansion for the 2D Dyson gas, J. Phys. A 39 (2006) 8933-8963, arXiv:hep-th/0601009

[73] S. Zelditch, Szegö kernels and a theorem of Tian, IMRN 1998 no. 6 (1998) 317-331, arXiv:math-ph/0002009.

[74] P. G. Zograf and L. A. Takhtadzhyan, A local index theorem for families of $\bar{\partial}$-operators on Riemann surfaces, Uspekhi Mat. Nauk 42 no. 6(258) (1987) 133-150 (Russian); English translation in Russian Math. Surveys 42 (1987) 169-190. 